\title{
DNA damage contributes to age-associated differences in SARS-CoV-2 infection
}

\section{Rui Jin}

Beijing Institute of Biotechnology

\section{Chang Niu}

College of Life Sciences, Capital Normal University

\section{Fengyun Wu}

College of Life Sciences, Capital Normal University

\section{Sixin Zhou}

Chinese PLA General Hospital

\section{Tao Han}

Chinese PLA General Hospital

\section{Zhe Zhang}

Beijing Institute of Biotechnology, Beijing, 100850, China https://orcid.org/0000-0002-9303-8221

\section{Xiaona Zhang}

College of Life Sciences, Capital Normal University

\section{Shanrong Xu}

Anqing Normal University

Jun Zhang

Beijing Institute of Biotechnology https://orcid.org/0000-0002-9650-112X

\section{Xinyue Li}

Beijing Institute of Biotechnology

\section{Yinggui Wang}

Beijing Institute of Biotechnology

\section{Ting Gao}

Beijing Institute of Biotechnology

\section{Mengnan Yu}

Chinese PLA General Hospital

\section{Ruiguan Wang}

Chinese PLA General Hospital

\section{Changjian Zhang}

Chinese PLA General Hospital

\section{Jiangbo Li}

Beijing Institute of Biotechnology

Qi Liu 
Chinese PLA General Hospital

\section{Haijing Zhao}

Chinese PLA General Hospital

\section{Lingmei Qin}

Beijing Institute of Biotechnology

\section{Yufang Li}

College of Life Sciences, Capital Normal University

\section{Peng Yang}

the 967th Hospital of Chinese PLA

\section{Shen Tian}

College of Life Sciences, Capital Normal University

\section{Duoqi Zhou}

Anqing Normal University

\section{Wei Chen}

Laboratory of Vaccine and Antibody Engineering, Beijing Institute of Biotechnology, Beijing, China https://orcid.org/0000-0001-5805-2469

\section{Qinong Ye}

Beijing Institute of Technology https://orcid.org/0000-0001-8032-8919

\section{Cheng Cao}

Beijing Institute of Biotechnology

\section{Long Cheng ( $\square$ biolongcheng@outlook.com )}

Beijing Institute of Biotechnology https://orcid.org/0000-0002-4752-1298

\section{Biological Sciences - Article}

Keywords: SARS-CoV-2, COVID-19, coronavirus disease, disease severity, mortality

Posted Date: April 22nd, 2021

DOI: https://doi.org/10.21203/rs.3.rs-301544/v2

License: (9) This work is licensed under a Creative Commons Attribution 4.0 International License. Read Full License 
DNA damage contributes to age-associated differences in SARS-CoV-2 infection Rui Jin ${ }^{1 \#}$, Chang $\mathrm{Niu}^{2 \#}$, Fengyun $\mathrm{Wu}^{2 \#}$, Sixin Zhou ${ }^{3 \#}$, Tao Han ${ }^{4 \#}$, Zhe Zhang ${ }^{1 \#}$, Xiaona Zhang ${ }^{2}$, Shanrong $\mathrm{Xu}^{5}$, Jun Zhang ${ }^{1}$, Xinyue $\mathrm{Li}^{1}$, Yinggui Wang ${ }^{1}$, Ting Gao ${ }^{1}$, Mengnan $\mathrm{Yu}^{4}$, Ruiguan Wang ${ }^{6}$, Changjian Zhang ${ }^{7}$, Jiangbo $\mathrm{Li}^{1}$, Qi Liu ${ }^{7}$, Haijing Zhao ${ }^{7}$, Lingmei Qin ${ }^{1}$, Yufang $\mathrm{Li}^{2}$, Peng Yang ${ }^{8}$, Shen Tian ${ }^{2}$, Duoqi Zhou ${ }^{5}$, Wei Chen ${ }^{1 *}$, Qinong $\mathrm{Ye}^{1^{*}}$, Cheng $\mathrm{Cao}^{1 *}$, Long Cheng ${ }^{1 *}$

${ }^{1}$ Beijing Institute of Biotechnology, Beijing 100850, China. ${ }^{2}$ College of Life Sciences, Capital Normal University, Beijing 100048, China. ${ }^{3}$ Department of Surgery, ${ }^{4} \mathrm{BaYi}$ Children's Hospital, the Seventh Medical Center, ${ }^{6}$ Faculty of Hepato-Pancreato-Biliary Surgery, ${ }^{7}$ Department of Cardiology, the Sixth Medical Center, Chinese PLA General Hospital, Beijing 100853, China. ${ }^{5}$ School of Life Science, Anqing Normal University, Anqing, Anhui 246133, China. ${ }^{8}$ Department of Clinical Laboratory, the 967th Hospital of Chinese PLA, Dalian, Liaoning 116000, China.

${ }^{\#}$ These authors contributed equally to this work

*Corresponding author. Email: biolongcheng@,outlook.com (L.C.); cao_c@,sohu.com (C.C.); yeqn66@yahoo.com (Q.Y.); cw0226@,foxmail.com (W.C.) 
Coronavirus disease 2019 (COVID-19), caused by coronavirus SARS-CoV-2, is known to disproportionately affect older individuals ${ }^{1,2}$. How aging processes affect the disease progression remains largely unknown. Here we found that DNA damage, one of the major causes of aging ${ }^{3}$, promoted susceptibility to SARS-CoV-2 infection in cells and intestinal organoids. SARS-CoV-2 entry was facilitated by DNA damage caused by telomere attrition or extrinsic genotoxic stress and hampered by inhibition of DNA damage response (DDR). Mechanistic analysis revealed that DDR increased expression of ACE2, the receptor of SARS-CoV-2, by activation of transcription factor c-Jun in vitro and in vivo. Expression of ACE2 was elevated in the older tissues and positively correlated with $\gamma \mathrm{H} 2 \mathrm{Ax}$ and phosphorylated c-Jun (p-c-Jun). Finally, targeting DNA damage by increasing the DNA repair capacity, alleviated cell susceptibility to SARS$\mathrm{CoV}-2$. Our data provide insights into the age-associated differences in SARS-CoV-2 infection and a novel target for anti-viral intervention.

SARS-CoV-2, the coronavirus responsible for the current COVID-19 pandemic, primarily infiltrates cells through the receptor ACE2. This membrane protein is also the receptor of SARS-CoV which led to an outbreak in 20034. COVID-19 disproportionately affects older individuals, who are more likely to develop severe symptoms and experience higher mortality ${ }^{1,2}$. Many possible reasons underlie these age-associated differences, including different cell susceptibility to viruses and different immune response and capacity against viral infection ${ }^{5,6}$. An inherent aspect of the aging process is the accumulation of DNA damage over time, which can be detected by $\gamma \mathrm{H} 2 \mathrm{Ax}$ staining ${ }^{3,7,8}$. Although most DNA lesions arising from extrinsic or intrinsic damage are quickly repaired, a very small number of highly toxic lesions can persist and accumulate, especially DNA damage occurring at telomeres, which is caused by telomere attrition upon cell division or genotoxic stress ${ }^{9,10}$. Another reason for the accumulation of DNA damage is the decline in DNA repair capacity arising from changes in the expression or activity of molecules involved in DNA repair ${ }^{11}$. Moreover, DNA damage was one of the primary causes of aging ${ }^{12-14}$. Numerous premature aging diseases, such as Werner syndrome and Bloom syndrome, are the consequences of 
increased accumulation of DNA damage caused by mutations in genes involved in the maintenance of genomic stability ${ }^{15}$.

To address whether telomere dysfunction contribute to cell susceptibility to SARSCoV-2, we first generated Calu-3 cells with short telomeres by knockout of TERC, a core component of telomerase which compensates telomere repeats during each cell cycle in stem cells and most cancer cells (Fig. S1a). TERC knockout inhibited telomerase activity and long-term culture of the cells resulted in telomere attrition, telomere damage and subsequent cellular senescence (Fig. S1b-d, Fig. 1a). Notably, cells with shorter telomeres were more susceptible to SARS-CoV-2 pseudovirus infection than cells with normal telomere length (Fig. 1a). To detect whether telomere attrition or subsequent telomere damage rendered viral entry, TPP1 $\triangle \mathrm{RD}$ and POT1 $\triangle \mathrm{OB}$, two mutants of shelterin components, were transiently transfected into Caco-2 cells to induce telomere damage without telomere attrition ${ }^{16}$. We found that cells expressing the mutants also displayed increased susceptibility to SARS-CoV-2 pseudovirus (Fig. S1e). These observations suggest that DNA damage at telomeres caused by telomere dysfunction may facilitate SARS-CoV-2 infection. Next, to explore whether DNA damages caused by genotoxic stress promotes viral entry, Calu-3-Rluc cells (Calu-3 cells stably expressing Renilla-luciferase) were treated with gamma ionizing radiation (IR) and subsequently infected with lentiviral particles pseudotyped with the $\mathrm{S}$ protein of SARS-CoV-2, SARS-CoV, MERS-CoV, or VSV-G protein. Cell susceptibility to SARS-CoV-2 and SARS-CoV pseudovirus was increased after IR treatment, but infectivity was decreased when the DNA damage was repaired at 7 days after the $1 \mathrm{~Gy}$ IR treatment (Fig. 1b and Fig. S2a). In contrast, infection by MERS-CoV and VSV-G pseudovirus was unaffected by IR treatment. Similar results were observed in Caco-2Rluc cells (Fig. S2b). Additionally, cell susceptibility was not affected by nucleolar stress, another stress also contributed to cell senescence and aging without induction of DNA damage ${ }^{17}$, through knockdown of PES1 or WDR12 (Fig. S2c). Collectively, these results indicate that DNA damage, caused by either telomere dysfunction or IR stress, can increase cell susceptibility to SARS-CoV-2. 
To ascertain whether DNA damage per se or the accompanied induction of reactive oxygen species leads to increased SARS-CoV-2 infection, Caco-2-Rluc cells were treated with the DDR inhibitor caffeine, KU55933 or VE-822. These inhibitors dramatically inhibited the DDR and SARS-CoV-2 pseudovirus entry after IR (Fig. 1c). Additionally, the DNA damage induced by other genotoxic agents, including etoposide, UV and $\mathrm{H} 2 \mathrm{O} 2$, promoted SARS-CoV-2 pseudovirus infection and was inhibited by caffeine (Fig. S2d-f). Notably, knockdown of ATM and ATR, two critical mediators of the DDR, also reduced viral entry (Fig. S2g). These results highlight the critical role of the DDR in DNA damage induced SARS-CoV-2 infection. Relatedly, because the application of intestinal organoids has been validated as a promising model of SARSCoV-2 infection ${ }^{18,19}$, we isolated intestinal stem cells from a neonate (Line 0 ) and a 55year-old woman (Line 46) and cultured them as organoids. Consistent with the cell culture results, the susceptibility of the organoids to SARS-CoV-2 pseudovirus was enhanced by IR treatment and inhibited by caffeine (Fig. 1d). We next explored the role of DNA damage in cell susceptibility to authentic SARS-CoV-2 virus infection, and found that authentic viral entry was also enhanced by IR and inhibited by caffeine in Caco-2 cells (Fig. 1e). Therefore, these results indicate that DNA damage and the subsequent DDR facilitate SARS-CoV-2 infection.

Because the entry of SARS-CoV-2 and SARS-CoV (but not VSV and MERS-CoV) was enhanced by DNA damage, we hypothesize that DNA damage may regulate the expression of ACE2, the receptor of SARS-CoV-2 and SARS-CoV. As anticipated, IR treatment markedly increased the expression of ACE2, but not TMPRSS2, another protein involved in SARS-CoV-2 entry, or DPP4, the receptor of MERS-CoV (Fig. 2a, Fig. S3a). Moreover, ACE2 expression was induced by DNA damage from various stimuli and impaired after inhibiting the DDR pathway by treatment with si-ATM, siATR or caffeine (Fig. 2b, Fig. S3b-d). These observations suggested that DNA damage and subsequent DDR upregulate ACE2 expression, which explained the previous findings of virus entry. Further analysis demonstrated that DNA damage induced ACE2 
expression at the transcriptional level (Fig. S4a). Meanwhile, DNA damage increased the expression of IFN- $\beta$, one component of interferon SASP (Senescence-associated secretory phenotype), and its target genes, including ISG15 and OAS1 ${ }^{20}$. Considering the type-I interferon increases ACE2 expression through Stat $1^{21}$, we next examined the role of Stat1 in this process. Knockdown of Stat 1 did not alter ACE2 expression in the presence and absence of IR (Fig. S4b), which could be explained by a recent finding showing that interferons stimulated the expression of a truncated form of ACE2, which could not mediate virus entry ${ }^{22}$. We next screened transcription factors that were predicted to bind to the ACE2 promoter according to the JASPR database. Knockdown of c-Jun, but not other transcription factors, significantly inhibited ACE2 expression (Fig. S4c, Fig. 2c). c-Jun knockdown also inhibited cell susceptibility to SARS-CoV-2 pseudovirus and authentic virus (Fig. S4d, Fig. 2d). Additionally, the total and phosphorylated c-Jun levels were also upregulated by IR and inhibited by DDR inhibitor (Fig. 2c, Fig. S4e, Fig. 2e), which was coincided with previously reported ${ }^{23}$. Beside IR-induced DNA damage, telomere damage caused by TERC knockout or transfection of mutants of shelterin components also increased expression of ACE2 and c-Jun (Fig. 2f, Fig. S4f). To further confirm the regulation of c-Jun on ACE2, we performed a ChIP assay and verified that p-c-Jun could bind to the ACE2 promoter region and a reported promoter region of ${\mathrm{c}-\mathrm{Fos}^{24}}^{24}$ but not to a distal region of ACE2 (Fig. 2g). Taken together, these observations provided in vitro evidences that DNA damage facilitates SARS-CoV-2 entry through upregulate the expression of ACE2 by increasing c-Jun expression. Next, we tested the regulatory mechanism in human intestinal organoids. IR treatment promoted the expression of ACE2 and p-c-Jun in neonate organoid (Line 0) and inhibition of DDR by caffeine reduced ACE2 and p-cJun expression in aged organoid (Line 46) (Fig. 2h). Additionally, to explore the effect of DNA damage on ACE2 expression in vivo, 8-week-old mice were treated with or without IR and ACE2 expression was analyzed in lung tissues. Bronchial epithelial cells reportedly express high levels of ACE2 ${ }^{21}$. IR treatment increased the expression of 
ACE2 and p-c-Jun (Fig. 2i). Thus, these observations suggest that DNA damage increases ACE2 expression through the activation of c-Jun, both in vitro and in vivo.

To approach the clinical evidence of the role of DNA damage in SARS-CoV-2 infection during aging, we analyzed the expression of ACE2, $\gamma \mathrm{H} 2 \mathrm{Ax}$ and p-c-Jun in old and young tissues from human intestinal and mouse lung. The expression of ACE2, $\gamma \mathrm{H} 2 \mathrm{Ax}$ and p-c-Jun were increased in older human small intestine tissues (Fig. 3a, b). Elevated ACE2 expression was also detected in older mouse bronchial tissues (Fig. S5a, b). Additionally, ACE2 expression positively correlated with $\gamma \mathrm{H} 2 \mathrm{Ax}$ and p-c-Jun expression in human intestinal tissues (Fig. 3c-e). The nasal cavity is typically the primary site of SARS-CoV-2 infection and thus plays an important role in virus transmission ${ }^{25}$. Thus, we compared the expression of c-Jun in $\mathrm{ACE}^{+}$and ACE2- human nasal ciliated 2 cells, which have been identified to express highest level of ACE2 in nasal cavity ${ }^{26}$, using published scRNA-seq data ${ }^{27}$. c-Jun expression was significantly upregulated in $\mathrm{ACE}^{+}$cells compared with ACE2- cells (Fig. 3f). Additionally, a Pathway Interaction Database (PID) analysis of 73 upregulated genes in $\mathrm{ACE}^{+}$ciliated 2 cells indicated the AP-1 and ATF2 signaling pathways were the most significantly enriched (Fig. 3g, Supplementary Table 1). Consistently, these pathways could be activated by DNA damage and c-Jun plays a major role in the signal transduction ${ }^{23,28}$.

The decline of capacity to repair DNA damage with age strongly contributes to the age-associated accumulation of DNA damage ${ }^{8}$. To mitigate the increased cell susceptibility to SARS-CoV-2 infection caused by DNA damage, we treated cells with two well-examined compounds, NMN and MDL-800, in an attempt to increase the DNA repair capacity ${ }^{29,30}$. NMN and MDL-800 both inhibited the expression of $\gamma \mathrm{H} 2 \mathrm{Ax}$ in Caco-2 cells, indicating the repair of DNA damage. Importantly, these compounds also inhibited cell susceptibility to SARS-CoV-2 pseudovirus and the expression of ACE2 and c-Jun (Fig. 4a). Furthermore, organoids isolated from aged woman were used to explore the function of MDL-800. NMN was not included in the experiment 
because it can inhibit organoid differentiation. As expected, MDL-800 reduced SARSCoV-2 pseudovirus infection, as well as ACE2, $\gamma \mathrm{H} 2 \mathrm{Ax}$ and c-Jun expression. (Fig. 4b).

In conclusion, these findings reveal that DNA damage, caused by either telomere dysfunction or extracellular stimuli, facilitates SARS-CoV-2 infection by increasing the expression of ACE2. Although the detailed mechanisms remain unclear, we found that c-Jun is required for DNA damage induced ACE2 expression and viral entry. c-Jun has been reported to be activated by DNA damage and upregulated in older human and mouse tissues ${ }^{31,32}$. Thus, we propose a DNA damage/c-Jun/ACE2 regulatory mechanism to explain the age-associated severity and outcome of COVID-19, although other characteristics and alterations of aging can not be excluded. Other factors involved in COVID-19 progression, such as smoking and obesity, are also associated with ACE2 upregulation ${ }^{33-35}$. Moreover, both smoking and obesity could cause, or at least associate with the elevated level of DNA damage ${ }^{36,37}$, which suggests the potential role of DNA damage in the smoking- and obesity-related risk of COVID-19. In addition, a positive correlation between DNA damage and the expression levels of p-c-Jun and ACE2 in human tissues was identified in the current study, which provide a clinical evidence of the regulatory mechanism. Finally, we found that targeting DNA damage, either by using inhibitors to inhibit DDR or by increasing the DNA repair capacity through treatment with NMN and MDL-800, reduced cell susceptibility to SARS-CoV2 infection in cell lines and organoids. Interestingly, an NMN cocktail has been applied to treat older people with complicated SARS-CoV-2 infections and has resulted in rapid and dramatic clinical and laboratory improvement ${ }^{38}$. Although the function of NMN in COVID-19 has not been proven conclusively, targeting DNA damage may constitute a new approach for COVID-19 therapies, especially in older adults.

\section{References}

1 O'Driscoll, M. et al. Age-specific mortality and immunity patterns of SARSCoV-2. Nature 590, 140-145, doi:10.1038/s41586-020-2918-0 (2020).

2 Williamson, E. J. et al. Factors associated with COVID-19-related death using OpenSAFELY. Nature 584, 430-436, doi:10.1038/s41586-020-2521-4 (2020). 
3 Lopez-Otin, C., Blasco, M. A., Partridge, L., Serrano, M. \& Kroemer, G. The hallmarks of aging. Cell 153, 1194-1217, doi:10.1016/j.cell.2013.05.039 (2013).

4 shi, z. 1. A pneumonia outbreak associated with a new coronavirus of probable bat origin. doi:10.1038/s41586-020-2012-7 (2020).

5 Mueller, A. L., McNamara, M. S. \& Sinclair, D. A. Why does COVID-19 disproportionately affect older people? Aging (Albany NY) 12, 9959-9981, doi:10.18632/aging.103344 (2020).

6 Wang, A. et al. Single-cell multiomic profiling of human lungs reveals celltype-specific and age-dynamic control of SARS-CoV2 host genes. eLife 9, doi:10.7554/eLife.62522 (2020).

7 Mah, L. J., El-Osta, A. \& Karagiannis, T. C. GammaH2AX as a molecular marker of aging and disease. Epigenetics 5, 129-136, doi:10.4161/epi.5.2.11080 (2010).

8 Sedelnikova, O. A. et al. Senescing human cells and ageing mice accumulate DNA lesions with unrepairable double-strand breaks. Nat. Cell Biol. 6, 168-170, doi:10.1038/ncb1095 (2004).

9 Hewitt, G. et al. Telomeres are favoured targets of a persistent DNA damage response in ageing and stress-induced senescence. Nature communications $\mathbf{3}$, 708, doi:10.1038/ncomms1708 (2012).

10 Fumagalli, M. et al. Telomeric DNA damage is irreparable and causes persistent DNA-damage-response activation. Nat. Cell Biol. 14, 355-365, doi:10.1038/ncb2466 (2012).

11 Di Micco, R., Krizhanovsky, V., Baker, D. \& d'Adda di Fagagna, F. Cellular senescence in ageing: from mechanisms to therapeutic opportunities. Nat. Rev. Mol. Cell Biol., doi:10.1038/s41580-020-00314-w (2020).

12 Moskalev, A. A. et al. The role of DNA damage and repair in aging through the prism of Koch-like criteria. Ageing research reviews 12, 661-684, doi:10.1016/j.arr.2012.02.001 (2013).

13 Gensler, H. L. \& Bernstein, H. DNA damage as the primary cause of aging. $Q$. Rev. Biol. 56, 279-303, doi:10.1086/412317 (1981).

14 White, R. R. \& Vijg, J. Do DNA Double-Strand Breaks Drive Aging? Mol. Cell 63, 729-738, doi:10.1016/j.molcel.2016.08.004 (2016).

15 Burtner, C. R. \& Kennedy, B. K. Progeria syndromes and ageing: what is the connection? Nat. Rev. Mol. Cell Biol. 11, 567-578, doi:10.1038/nrm2944 (2010).

16 Rai, R. \& Chang, S. Probing the Telomere Damage Response. Methods Mol. Biol. 1587, 133-138, doi:10.1007/978-1-4939-6892-3_13 (2017).

17 Nishimura, K. et al. Perturbation of ribosome biogenesis drives cells into senescence through 5S RNP-mediated p53 activation. Cell reports 10, 13101323, doi:10.1016/j.celrep.2015.01.055 (2015).

18 Zhou, J. et al. Infection of bat and human intestinal organoids by SARS-CoV-2. Nat. Med., doi:10.1038/s41591-020-0912-6 (2020).

19 Lamers, M. M. et al. SARS-CoV-2 productively infects human gut enterocytes. Science 369, 50-54, doi:10.1126/science.abc1669 (2020).

20 Nakao, M., Tanaka, H. \& Koga, T. Cellular Senescence Variation by Metabolic 
and Epigenomic Remodeling. Trends Cell Biol., doi:10.1016/j.tcb.2020.08.009 (2020).

21 Ziegler, C. G. K. et al. SARS-CoV-2 receptor ACE2 is an interferon-stimulated gene in human airway epithelial cells and is detected in specific cell subsets across tissues. Cell, doi:10.1016/j.cell.2020.04.035 (2020).

22 Onabajo, O. O. et al. Interferons and viruses induce a novel truncated ACE2 isoform and not the full-length SARS-CoV-2 receptor. Nat. Genet. 52, 12831293, doi:10.1038/s41588-020-00731-9 (2020).

23 Karin, M. The regulation of AP-1 activity by mitogen-activated protein kinases. J. Biol. Chem. 270, 16483-16486, doi:10.1074/jbc.270.28.16483 (1995).

24 Hayakawa, J. et al. Identification of promoters bound by c-Jun/ATF2 during rapid large-scale gene activation following genotoxic stress. Mol. Cell 16, 521535, doi:10.1016/j.molcel.2004.10.024 (2004).

25 Gallo, O., Locatello, L. G., Mazzoni, A., Novelli, L. \& Annunziato, F. The central role of the nasal microenvironment in the transmission, modulation, and clinical progression of SARS-CoV-2 infection. Mucosal Immunol., doi:10.1038/s41385-020-00359-2 (2020).

26 Hou, Y. J. et al. SARS-CoV-2 Reverse Genetics Reveals a Variable Infection Gradient in the Respiratory Tract. Cell 182, 429-446 e414, doi:10.1016/j.cell.2020.05.042 (2020).

27 Vieira Braga, F. A. et al. A cellular census of human lungs identifies novel cell states in health and in asthma. Nat. Med. 25, 1153-1163, doi:10.1038/s41591019-0468-5 (2019).

28 Christmann, M. \& Kaina, B. Transcriptional regulation of human DNA repair genes following genotoxic stress: trigger mechanisms, inducible responses and genotoxic adaptation. Nucleic Acids Res. 41, 8403-8420, doi:10.1093/nar/gkt635 (2013).

29 Chen, Y. et al. The SIRT6 activator MDL-800 improves genomic stability and pluripotency of old murine-derived iPS cells. Aging cell 19, e13185, doi:10.1111/acel.13185 (2020).

$30 \mathrm{Li}$, J. et al. A conserved $\mathrm{NAD}(+)$ binding pocket that regulates protein-protein interactions during aging. Science 355, 1312-1317, doi:10.1126/science.aad8242 (2017).

31 Biteau, B., Hochmuth, C. E. \& Jasper, H. JNK activity in somatic stem cells causes loss of tissue homeostasis in the aging Drosophila gut. Cell stem cell 3, 442-455, doi:10.1016/j.stem.2008.07.024 (2008).

32 Suh, Y. Age-specific changes in expression, activity, and activation of the c-Jun $\mathrm{NH}(2)$-terminal kinase and p38 mitogen-activated protein kinases by methyl methanesulfonate in rats. Mech. Ageing Dev. 122, 1797-1811, doi:10.1016/s0047-6374(01)00301-3 (2001).

33 Medina-Enriquez, M. M. et al. ACE2: the molecular doorway to SARS-CoV-2. Cell \& bioscience 10, 148, doi:10.1186/s13578-020-00519-8 (2020).

34 Smith, J. C. et al. Cigarette Smoke Exposure and Inflammatory Signaling Increase the Expression of the SARS-CoV-2 Receptor ACE2 in the Respiratory 
Tract. Dev. Cell 53, 514-529 e513, doi:10.1016/j.devcel.2020.05.012 (2020).

35 Sarver, D. C. \& Wong, G. W. Obesity alters Ace2 and Tmprss2 expression in lung, trachea, and esophagus in a sex-dependent manner: Implications for COVID-19. Biochem. Biophys. Res. Commun. 538, 92-96, doi:10.1016/j.bbrc.2020.10.066 (2021).

36 Hopkins, J. M. \& Evans, H. J. Cigarette smoke-induced DNA damage and lung cancer risks. Nature 283, 388-390, doi:10.1038/283388a0 (1980).

37 Usman, M. \& Volpi, E. V. DNA damage in obesity: Initiator, promoter and predictor of cancer. Mutat. Res. 778, 23-37, doi:10.1016/j.mrrev.2018.08.002 (2018).

38 Huizenga, Robert, Dramatic Clinical Improvement in Nine Consecutive Acutely Ill Elderly COVID-19 Patients Treated with a Nicotinamide Mononucleotide Cocktail: A Case Series (August 17, 2020). Available at SSRN: https://ssrn.com/abstract $=3677428$.

\section{Acknowledgments}

We thank Dr. Yanhua Li for kindly help with isolation and culture of intestinal organoid.

Funding: L.C. was supported by the National Natural Science Foundation (82072717) and Beijing Nova Program (Z191100001119020). Q.Y. was supported by National Natural Science Foundation (81630067 and 81930078 ). Z.Z. was supported by National Natural Science Foundation (31900671).

Author contributions: L.C., C.C. and Q.Y. conceived the study, designed the experiments and analyzed the data. W.C. and C.C. funded the study and designed the experiments of authentic viral infection. R.J. designed and performed the experiments aided by X.Z., S.X., X.L., Y.W., R.W., C.Z. and J.L. C.N. and F.W. isolated organoid and performed related experiments aided by Y.L. S.Z., T.H. and M.Y. provided the clinical samples and performed related analysis. Z.Z. performed experiment with authentic virus aided by J.Z. and T.G. Q.L., H.Z., L.Q and P. Y. performed IHC staining. L.C. drafted the manuscript and that was edited by S.T. and D.Z.

Competing interests: All authors declare that they have no competing interests.

Data availability: All data needed to evaluate the conclusions in the paper are present 
in the paper and/or the Supplementary Materials. Additional data related to this paper may be requested from the authors. 
Fig. 1

a

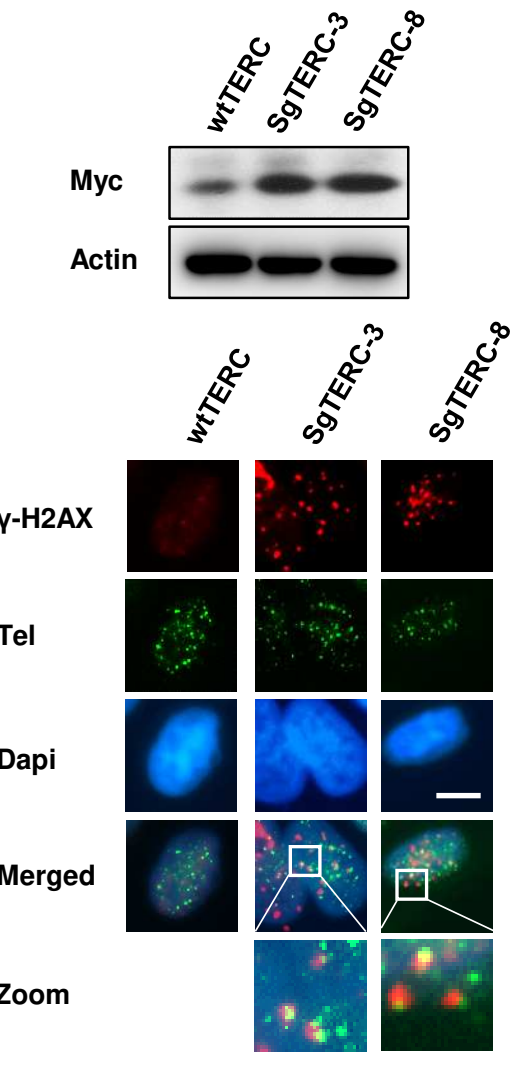

d

IR

Caffeine

Myc

Dapi

Phalloidin (647)

Merge

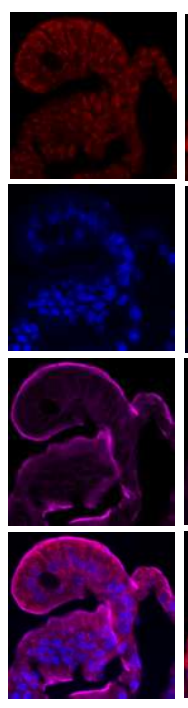

(neonate)

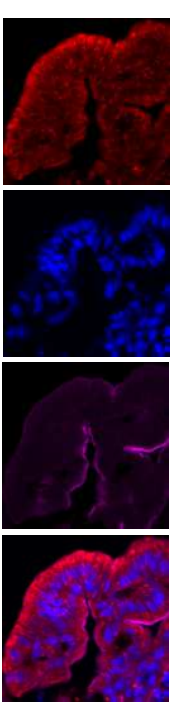

b
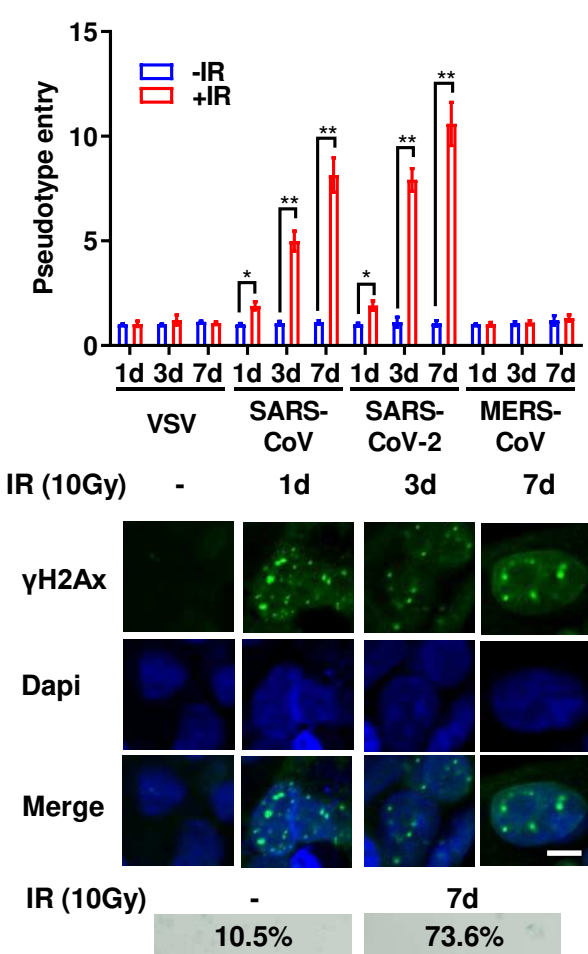

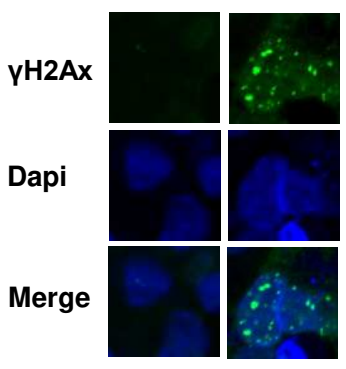

IR (10Gy)

SA- $\beta$-gal

Line 46

(55-year-old)

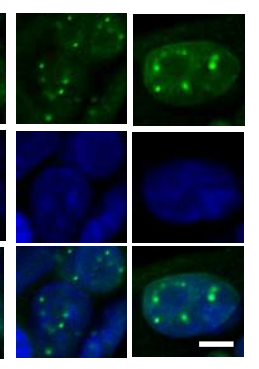

7d

$73.6 \%$

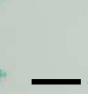

e
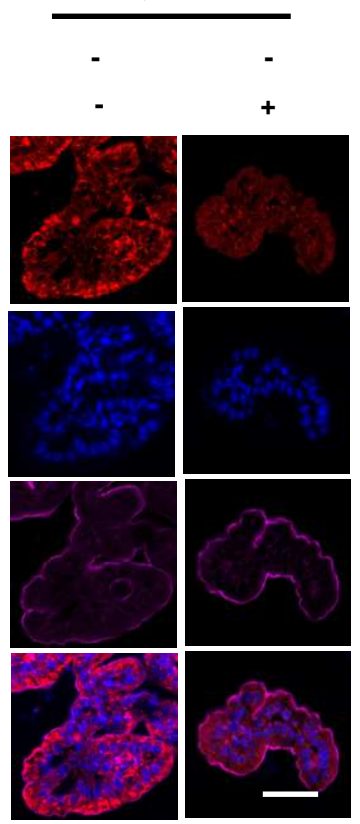

IR

Caffeine

Anti-S
C
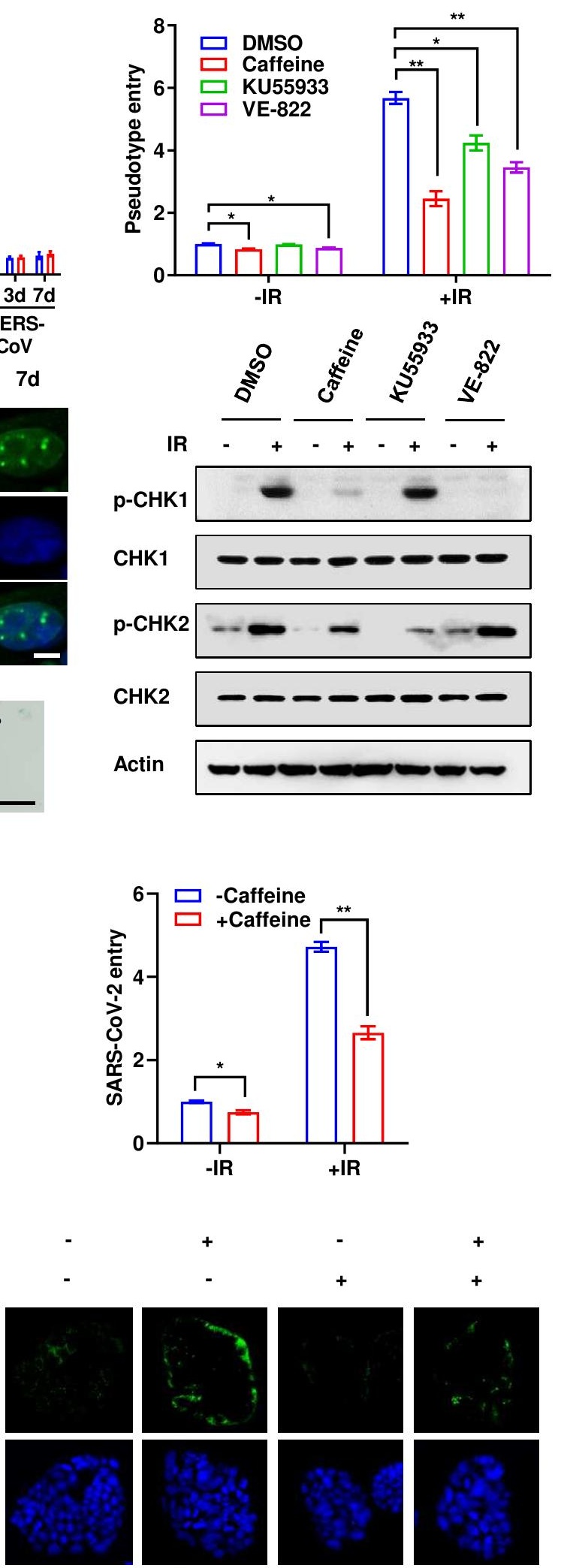

Dapi
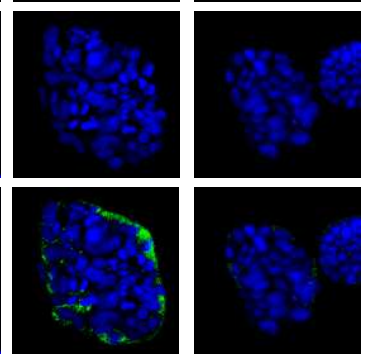
Fig. 1. DNA damage promotes SARS-CoV-2 entry. a, Monoclonal Calu-3 cells stably expressing sgTERC were infected with SARS-CoV-2 pseudovirus harbouring Myc-EGFP. Virus entry was analyzed by western blot at 3 days after infection using the indicated antibodies. Telomere damage was detected by immunostaining with an anti- $\mathrm{H} 2 \mathrm{Ax}$ antibody, followed by Tel-Fish. Scale bar, $10 \mu \mathrm{m}$. b, Calu-3-Rluc cells at various days after 10Gy IR treatment were infected with pseudotyped viruses harbouring a firefly luciferase reporter gene. Viral entry was calculated by dividing firefly-luciferase activity by renilla luciferase activity at 3 days after infection. DNA damage was detected by immunostaining with an anti-yH2Ax antibody. Scale bar, $10 \mu \mathrm{m}$. SA- $\beta$-gal staining was performed, and the results were quantified from $>5$ independent cell counts up to a total of at least 200 cells and are presented as the mean percentage of positive cells. Scale bar, $100 \mu \mathrm{m}$. c, Caco-2-Rluc cells were pretreated with caffeine $(1 \mathrm{mM})$, KU55933 $(10 \mu \mathrm{M})$ and VE-822 (100 nM) for 12h and then subjected to IR treatment (10Gy). Cells were infected with SARS-CoV-2 pseudovirus and analyzed as (b). d, Differentiated organoids isolated from a neonate (Line 0 ) and a 55-year-old woman (Line 46) were treated with IR (10Gy) or caffeine ( 1mM) and then infected with SARS-CoV-2 pseudovirus containing Myc-EGFP. Organoids were immunostained or detected by western blot using indicated antibodies at 3 day after infection. The EGFP signal was too weak to observe without immunostaining with Nyc antibody. Scale bar, $50 \mu \mathrm{m}$. e, Caco-2 cells treated with caffeine $(1 \mathrm{mM})$ and IR (10Gy), were incubated with authentic SARS-CoV-2 virus for $6 \mathrm{~h}$. The relative virus levels were detected by qRT-PCR analysis of SARS-CoV-2 N protein or immunostaining of SARS-CoV-2 S protein. Scale bar, $50 \mu \mathrm{m}$. All data are represented as the mean \pm SD of three (ac) or two (e) independent experiments. ${ }^{*} p<0.05,{ }^{* *} p<0.01,{ }^{* * *} p<0.001$. 
Fig. S1

a

SgTERC-3

SgTERC-3

wtTERC

GA GC C G A G A C A A G A T T C T G C T G TAG T

SgTERC-NAR

SgTERC-8

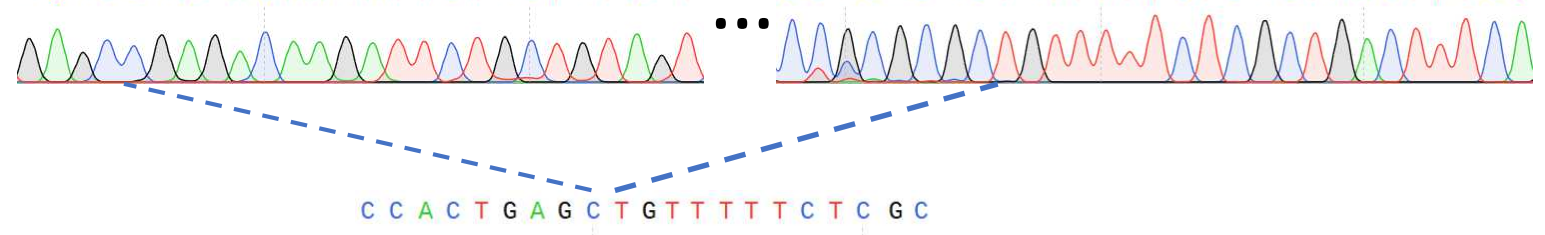

b

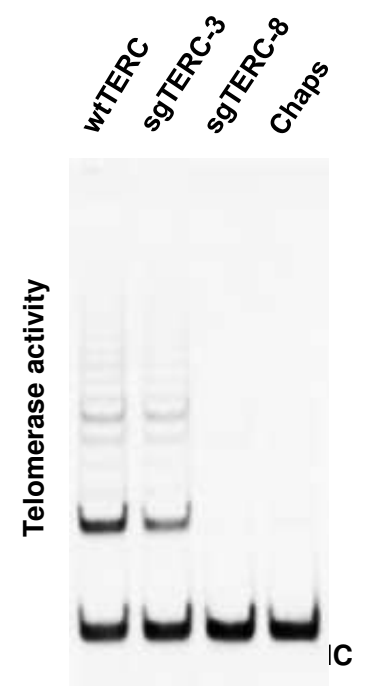

C

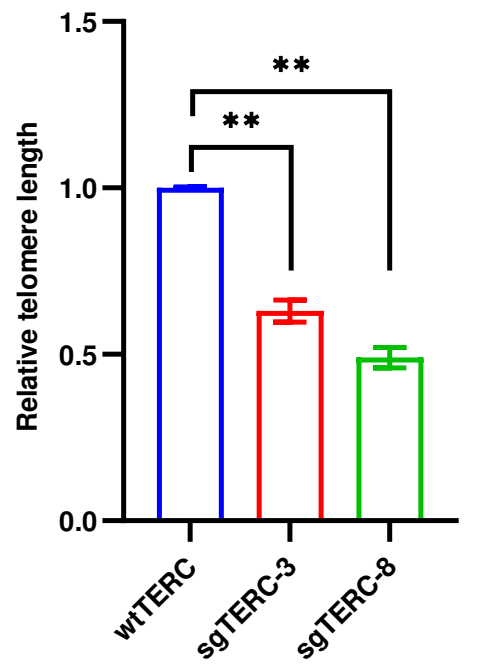

d e

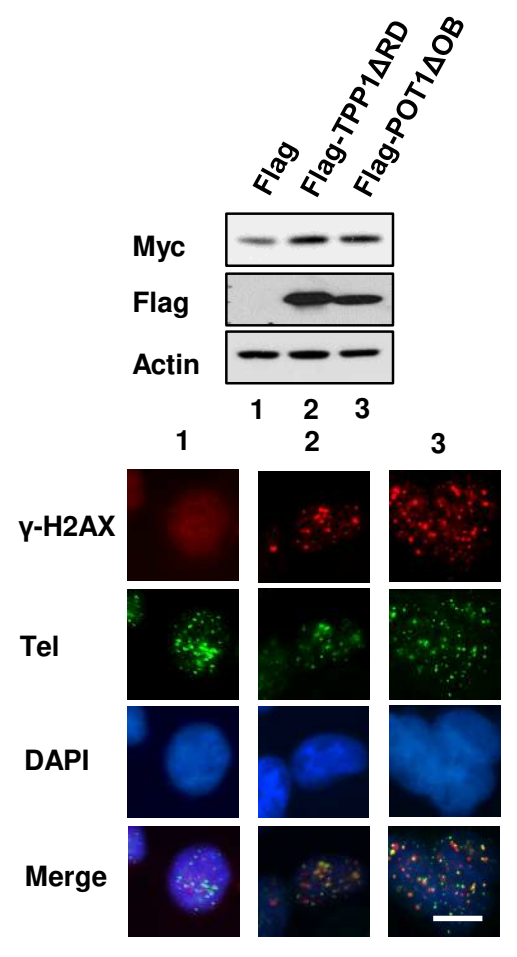

Fig. S1 Generation and identification of TERC knockout Calu-3 cells. a, Monoclonal Calu- 3 cells were identified by DNA sequencing. b. Telomerase activities of sgTERC-3 and sgTERC-8 cells were detected using TRAP assay. IC, internal control. c, Relative telomere length were examined in sgTERC-3 and sgTERC-8 cells. ${ }^{* *} p$ $<0.01$. d, SA- $\beta$-gal staining of Monoclonal Calu- 3 cells. SA- $\beta$-gal data were quantified from $>5$ independent cell counts up to a total of at least 200 cells and are presented as the mean percentage of positive cells. Scale bar, 100 $\mu \mathrm{m}$. e, Caco-2 cells stably expressing Flag-TPP1 $\triangle$ RD or Flag-POT1 $\triangle \mathrm{OB}$ were infected with SARS-CoV-2 pseudovirus containing Myc-EGFP. Viral entry was analyzed by detection of Myc and telomere damage was detected by staining with anti-yH2Ax antibody and Tel-Fish. Scale bar, $10 \mu \mathrm{m}$. 
Fig. S2

a

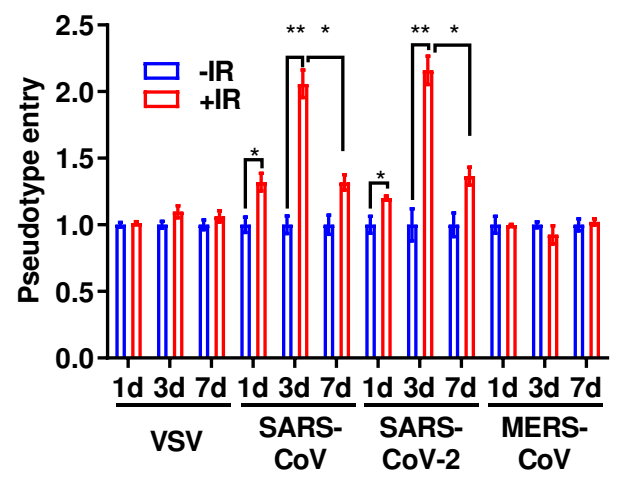

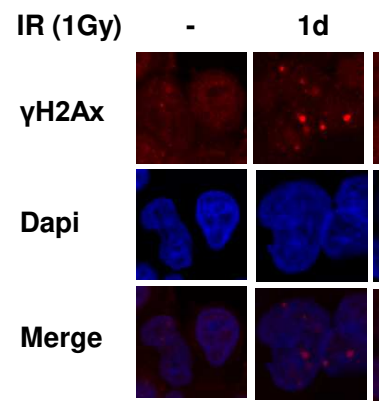

d

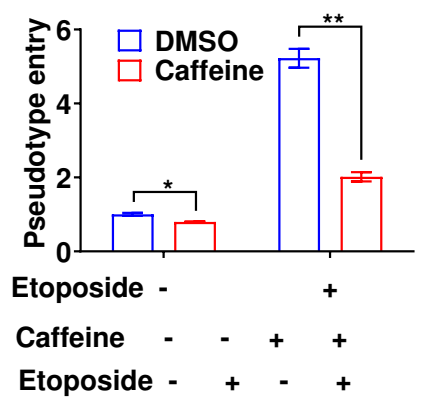

p-CHK2

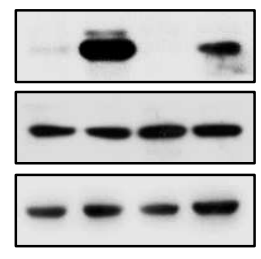

b
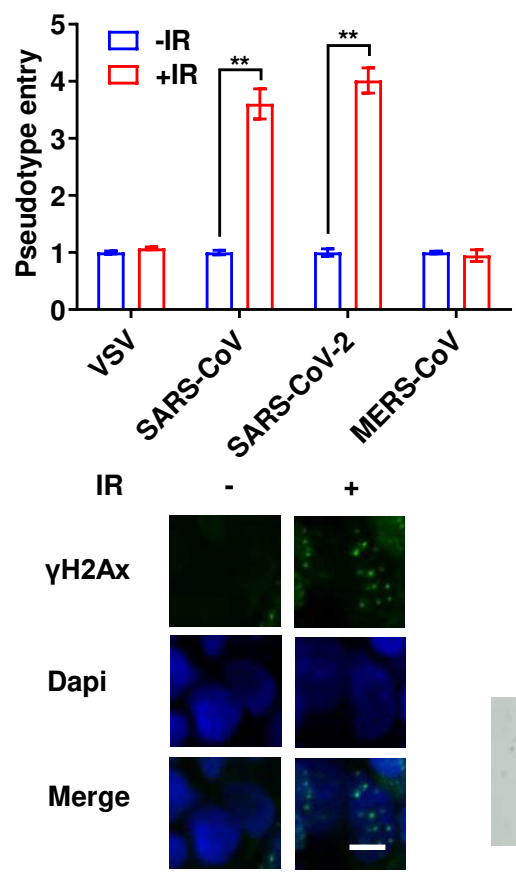

$\mathbf{f}$
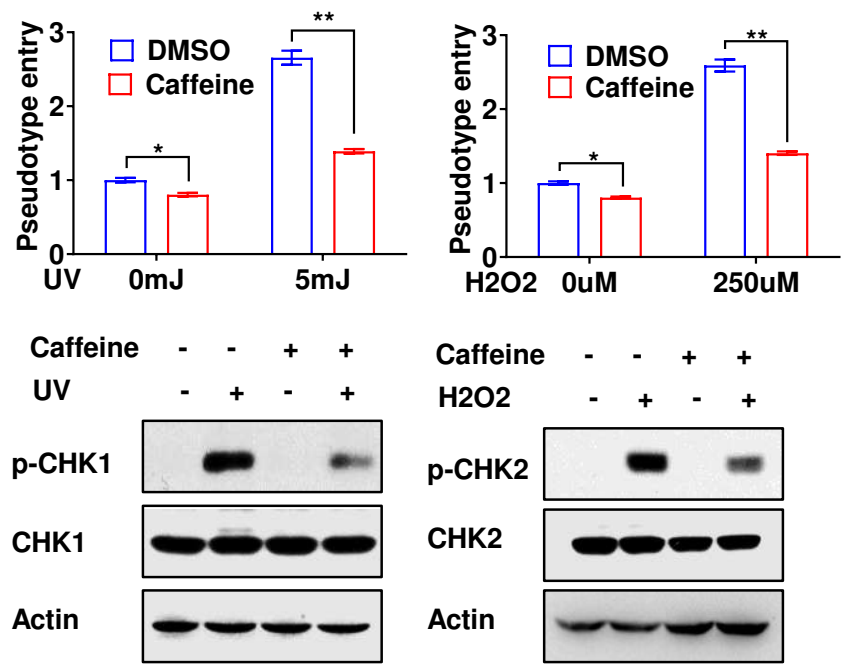

C

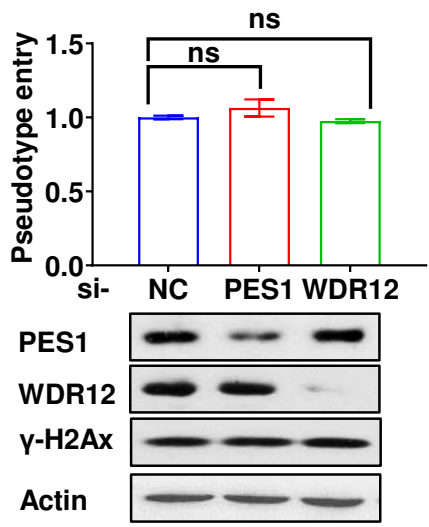

si-NC

si-PES1

Si-WDR12

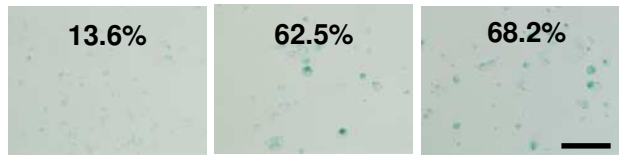

g
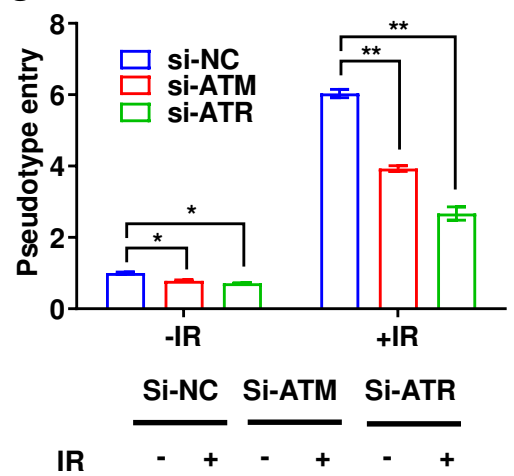

ATM

ATR

Actin

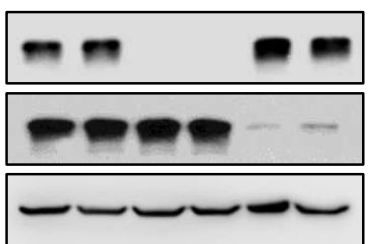

Fig. S2. DNA damage promotes SARS-CoV-2 entry dependent on DNA damage response. a, Calu-3-Rluc cells at various days after 1Gy IR treatment were subjected to pseudotyped virus infection. Viral entry was analyzed by luciferase activity at $72 \mathrm{~h}$ after infection. DNA damage was immunostained with anti-yH2Ax. Scale bars, $10 \mu \mathrm{m}$. b, Caco-2-Rluc cells at 3 days after 10Gy IR treatment were subjected to pseudotyped virus infection. Viral entry was analyzed by luciferase activity at $72 \mathrm{~h}$ after infection. DNA damage was detected by immunostaining with anti- $\mathrm{yH} 2 \mathrm{Ax}$ antibody. Scale bars, $10 \mu \mathrm{m}$. c, Caco-2-Rluc cells treated with PES1 or WDR12 siRNA were infected with SARS-CoV-2 pseudovirus. Viral entry and cell senescence were analyzed at $72 \mathrm{~h}$ after infection. Scale bar, $100 \mu \mathrm{m}$. d-f, Caco-2Rluc cells were pre-treated with caffeine ( $1 \mathrm{mM}$ ) for $12 \mathrm{~h}$ and then subjected to $500 \mathrm{nM}$ etoposide (d), UV (e) and H2O2 (f) treatment, followed by incubation with SARS-CoV-2 pseudoviruses at 3 days after treatment. Viral entry was analyzed by luciferase activity at $72 \mathrm{~h}$ after infection. g, Caco-2-Rluc cells transfected with ATM siRNA or ATR siRNA were incubated with SARS-CoV-2 pseudoviruses and viral entry was analyzed by luciferase activity at $72 \mathrm{~h}$ after infection. All data are represented as the mean \pm SD from three independent experiments. ${ }^{*} p<0.05,{ }^{\star *} p<0.01$. 
Fig. 2

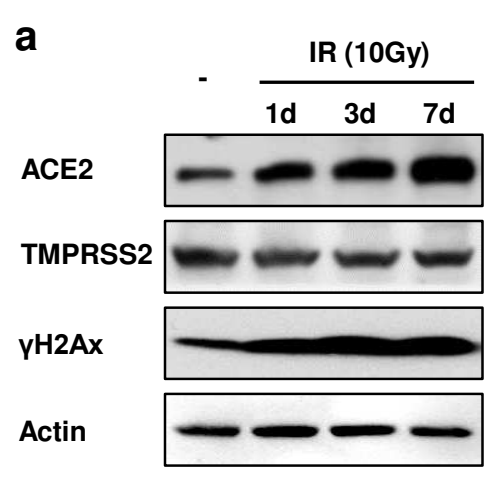

d

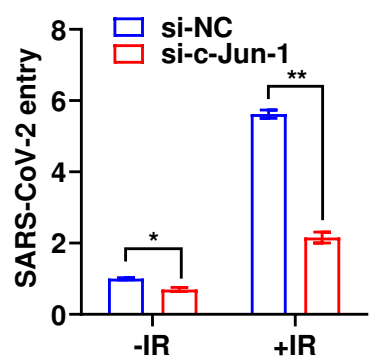

g

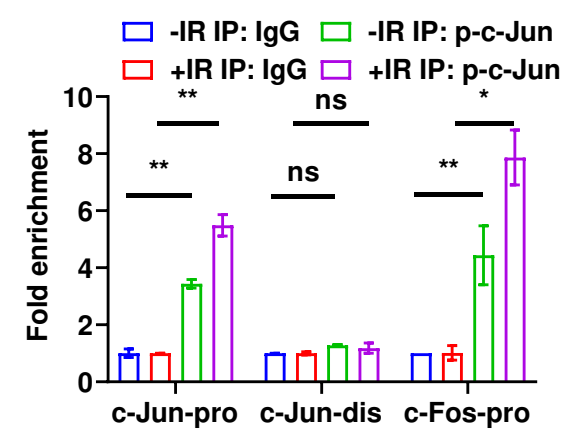

h

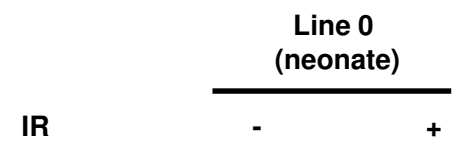

Caffeine

ACE2

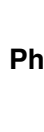

ACE2/

Phalloidin-647/

Dapi
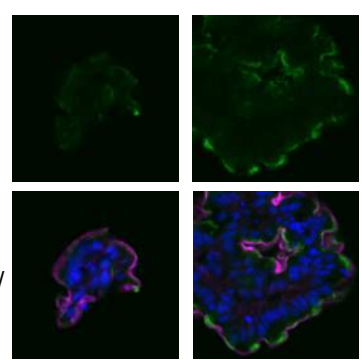

p-c-Jun

p-c-Jun/

Dapi b $\frac{\text { si-NC }}{-+} \frac{\text { si-ATM }}{-+} \frac{\text { si-ATR }}{-+}$

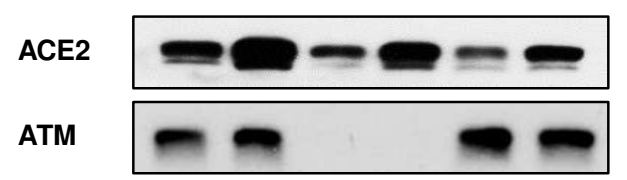

ATR

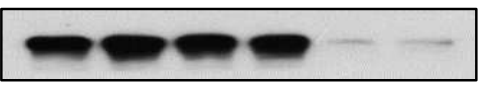

Actin

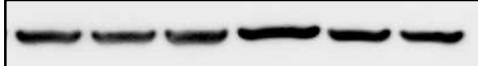

e

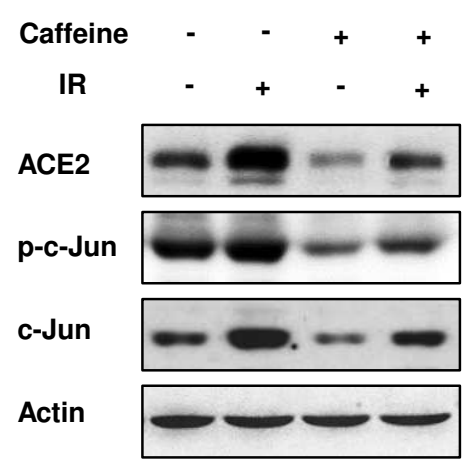

C

IR
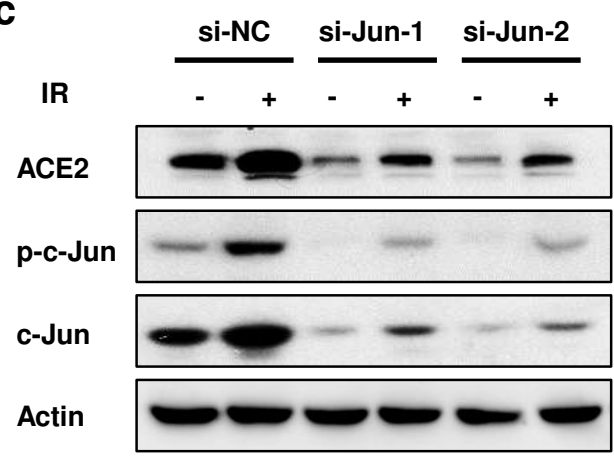

f

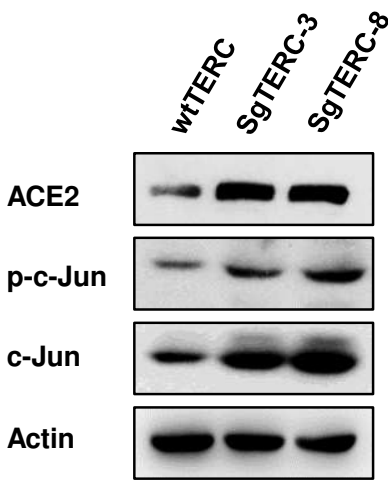

-IR

+IR

ACE2

p-c-Jun

Line 46

(55-year-old)

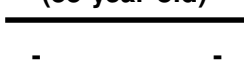

$+$

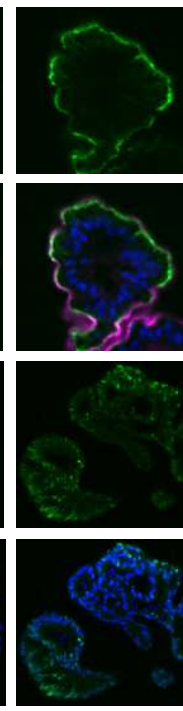

yH2Ax
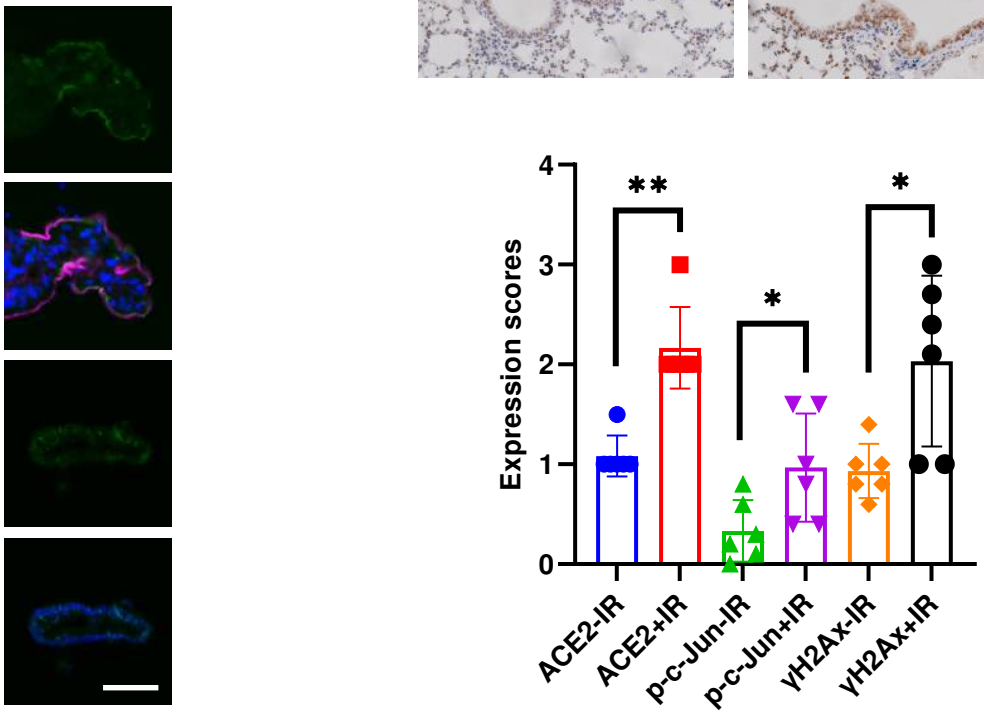
Fig. 2. DNA damage enhances ACE2 expression by activating c-Jun. a-b, Calu-3-Rluc cells at various days (a) or Caco-2 cells transfected with ATM or ATR siRNA at 3 days (b) after 10Gy IR treatment were subjected to western blot analysis using the indicated antibodies. c, Caco-2 cells after transfection with c-Jun siRNA were treated as in (b) and the expression of indicated genes were detected. d, Caco-2 cells were transfected with c-Jun siRNA 48h prior to infection with authentic SARS-CoV-2 virus for $6 \mathrm{~h}$ and then subjected to qRT-PCR analysis of the relative viral entry. e, Caco-2 cells were pre-treated with caffeine $(1 \mathrm{mM})$ for $12 \mathrm{~h}$ and then subjected to IR (10Gy). Western blot analysis was performed using the indicated antibodies at 3 days after IR. f, Monoclonal Calu-3 cells stably expressing sgTERC were subjected to western blot analysis using indicated antibodies. $\mathbf{g}$, ChIP analysis of the occupancy of pc-Jun on the indicated AP-1binding site of the C-Jun promoter (c-Jun pro) and distal regions (c-Jun dis) in Caco-2 cells at $3 \mathrm{~h}$ after 10 Gy IR treatment. The c-Fos promoter containing AP-1 binding site was included as a positive control. IgG, normal serum. Data shown are the mean \pm SD of triplicate measurements from three independent experiments. $\mathbf{h}$, Differentiated organoids treated with $I R(10 G y)$ or caffeine $(1 \mathrm{mM})$ were immunostained with the indicated antibodies. Scale bar, $50 \mu \mathrm{m}$. i, Expression of ACE2, p-c-Jun and $\mathrm{YH} 2 \mathrm{Ax}$ in 8-week-old mice 3 days after treated with $(n=6)$ or without $(n=6) 10 G y$ IR. Representative immunohistochemical staining of ACE2, $p$-c-Jun and yH2Ax was shown in the upper panel. Scale bar, $100 \mu \mathrm{m} .{ }^{*} p<0.05,{ }^{* *} p<0.01$. 
Fig. S3

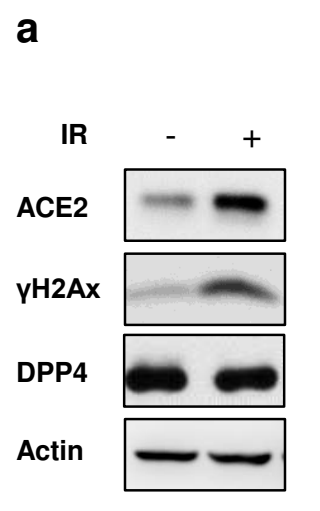

b

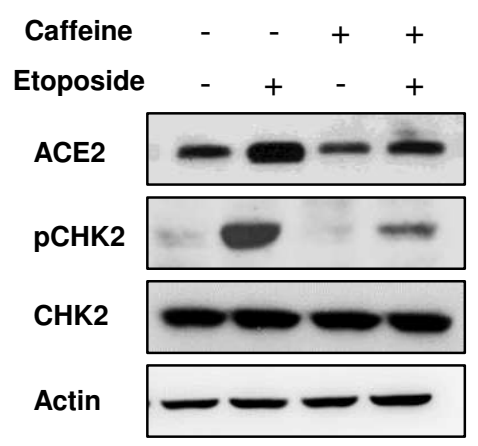

C

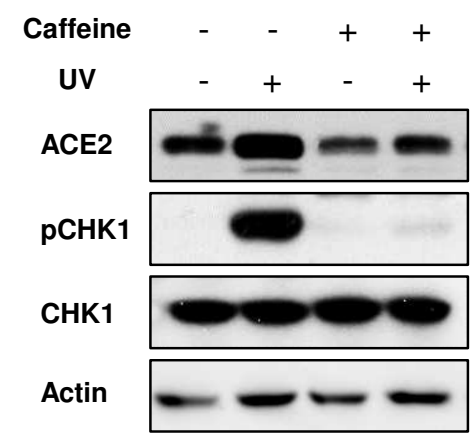

d

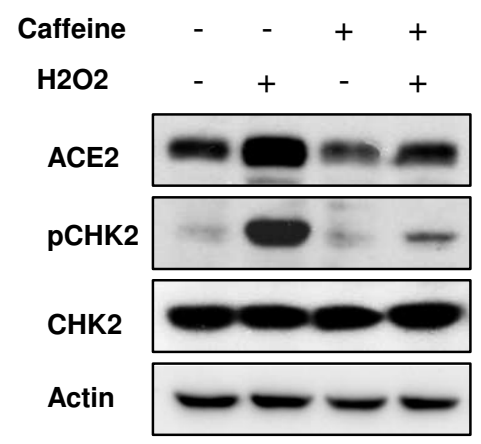

Fig. S3. DNA damage enhances expression of ACE2. a, Calu-3 cells at 3 days after 10Gy IR treatment were subjected to western blot analysis using indicated antibodies. $\mathbf{b}$-d, Caco-2 cells were pre-treated with caffeine $(1 \mathrm{mM})$ for $12 \mathrm{~h}$ and subjected to $500 \mathrm{nM}$ etoposide (b), $5 \mathrm{~mJ}$ UV (c) and $250 \mu \mathrm{M} \mathrm{H} 2 \mathrm{O} 2$ (d) treatment, followed by western blot analysis using indicated antibodies at 3 days after treatment. 
Fig. 54

a

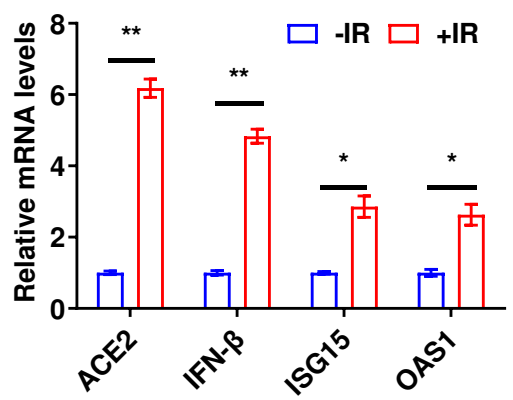

d

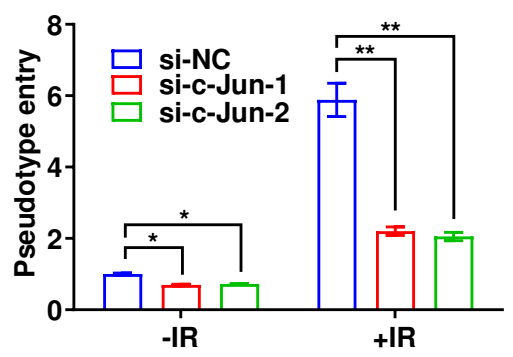

b

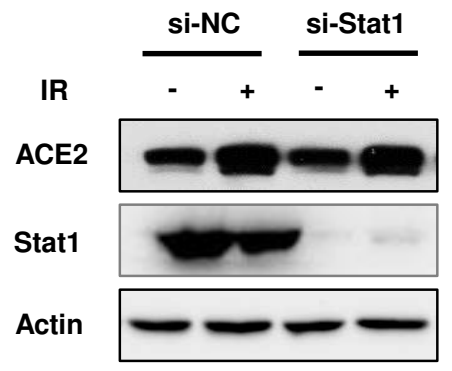

e

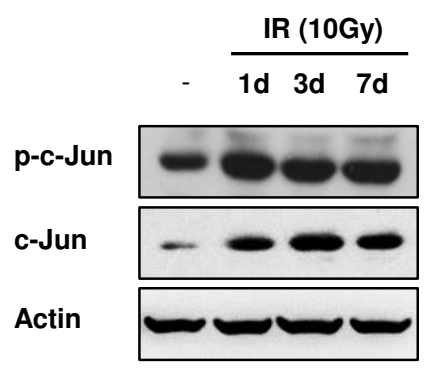

C

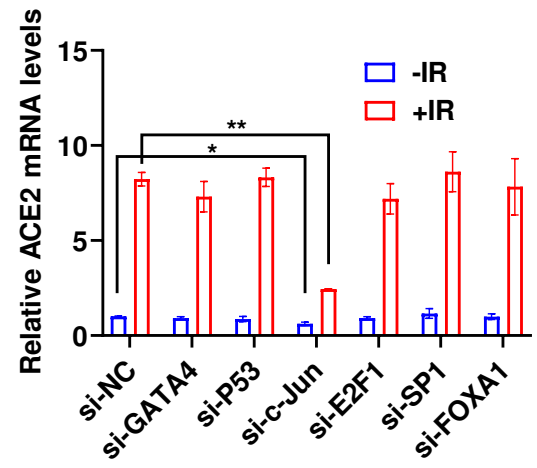

f

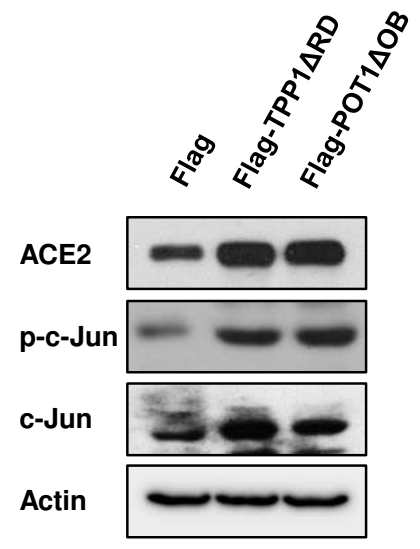

Fig. S4. DNA damage promotes ACE2 expression though activation of c-Jun. a, Calu-3-Rluc cells at 3 days after IR treatment (10Gy) were subjected to qRT-PCR analysis of mRNA levels of indicated genes. b, Caco-2 cells transfected with Stat1 siRNA were treated with 10Gy IR and then subjected to western blot analysis using the indicated antibodies at 3 days after IR treatment. c, Caco-2 cells transfected with indicated siRNA were treated with 10Gy IR and mRNA levels were detected at 48h after IR. d, Caco-2-Rluc cells transfected with c-Jun siRNAs were treated with 10Gy IR and subjected to SARS-CoV-2 pseudovirus infection at 3 days after IR. Viral entry was analyzed by luciferase activity at $72 \mathrm{~h}$ after infection. e, Calu-3-Rluc cells at different days after 10Gy IR treatment were subjected to western blot analysis using indicated antibodies. f, ACE2, phosphorylated and total c-Jun levels were detected in Caco-2 cells stably expressing Flag-TPP $1 \Delta$ RD or Flag-POT $1 \Delta$ OB. ${ }^{*} p<0.05,{ }^{* *} p<0.01$. 
Fig. 3

a

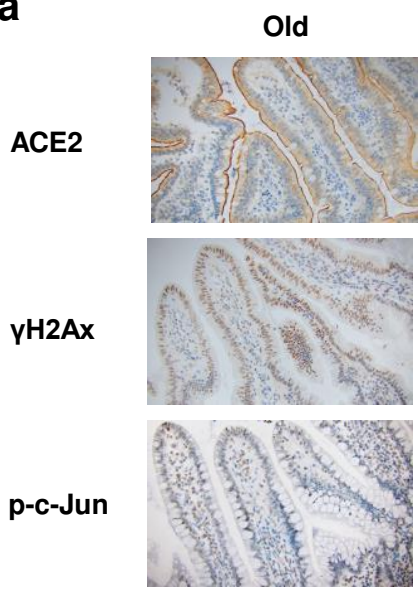

b
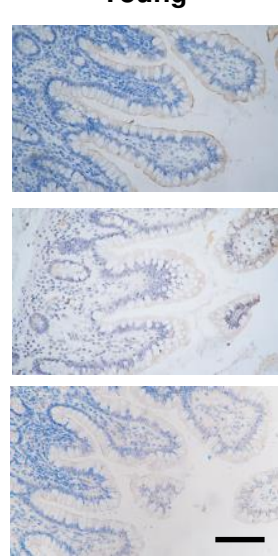

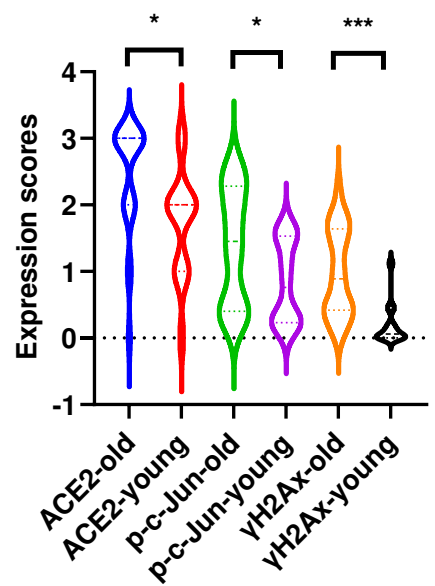

d

$r=0.3633$

$p=0.023$

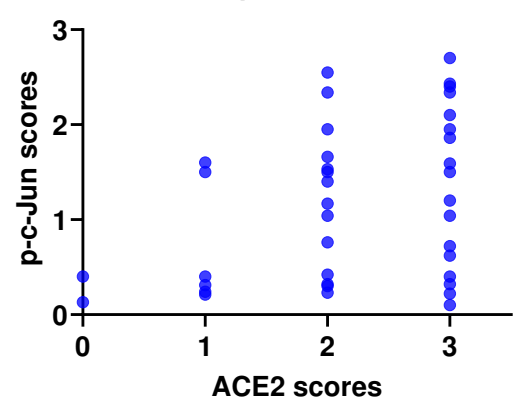

e
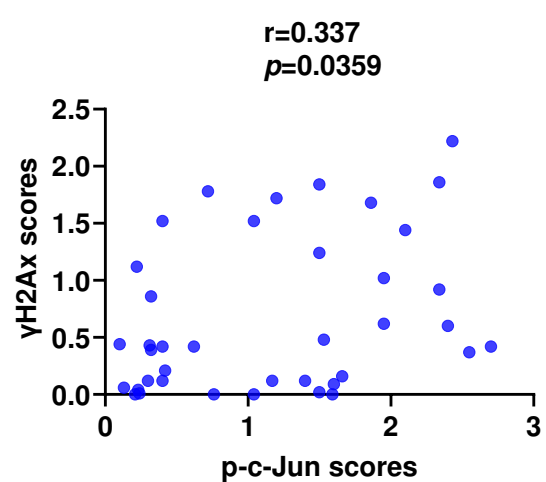

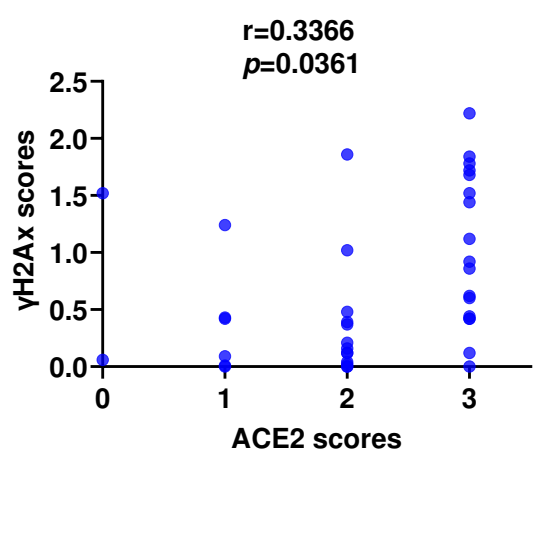

C

f

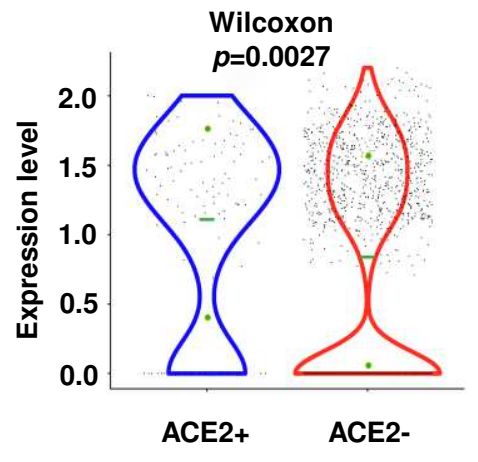

g

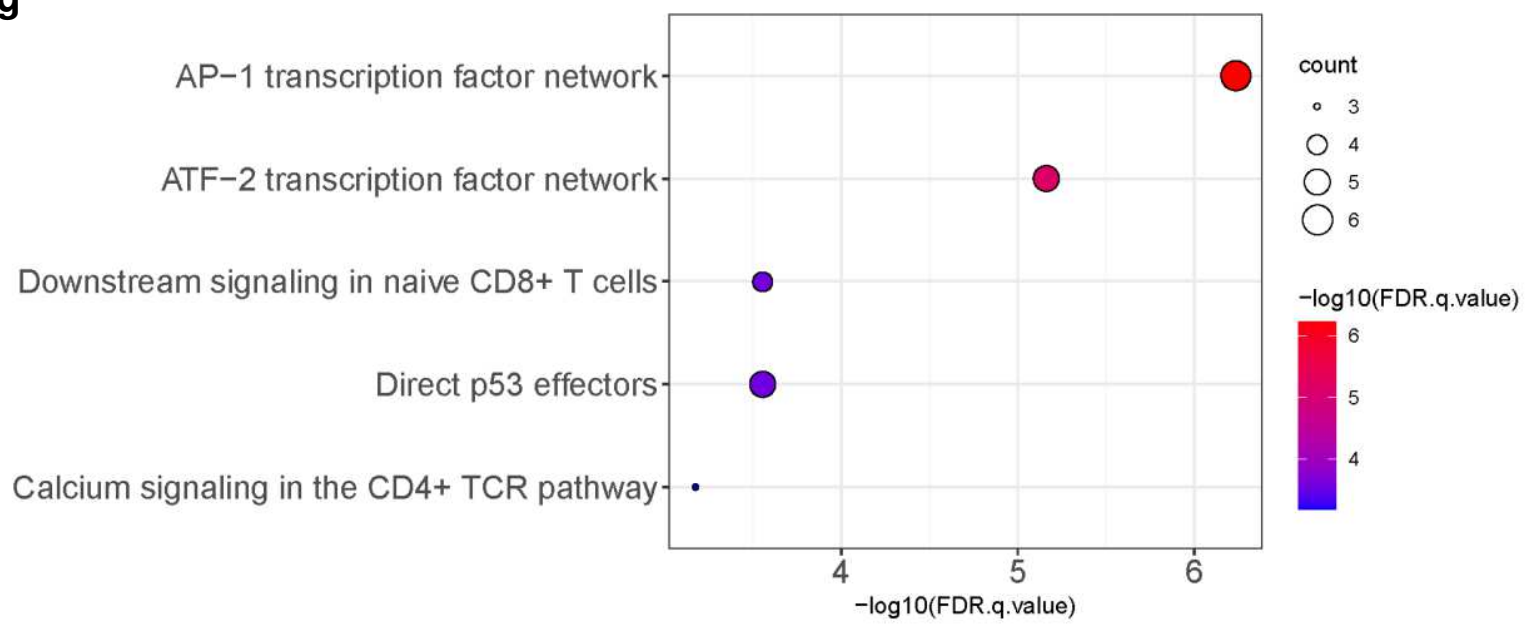


Fig. 3. Association between ACE2 and p-c-Jun and yH2Ax in human tissues. a, b, Expression of ACE2, p-c-Jun and $\mathrm{yH} 2 \mathrm{Ax}$ in old (60-73 years old, $\mathrm{n}=24$ ) and young (31-50 years old, $\mathrm{n}=15$ ) human intestinal tissues. Representative immunohistochemical staining of ACE2, p-c-Jun and yH2Ax is shown in (a). Scale bar, $100 \mu \mathrm{m}$. c-e, Correlations between expression of ACE2 and $\mathrm{yH} 2 \mathrm{Ax}$ (c), ACE2 and p-c-Jun (d), and p-c-Jun and $\mathrm{yH} 2 \mathrm{Ax}$ (e) were analyzed. f, Expression of c-Jun in ACE2+ and ACE2- nasal ciliated 2 cells. g, Pathway Interaction Database analysis of 73 up-regulated genes in ACE2+ nasal ciliated 2 cells. ${ }^{*} p<0.05,{ }^{* *} p<0.01,{ }^{* * *} p<0.001$. 
Fig. S5

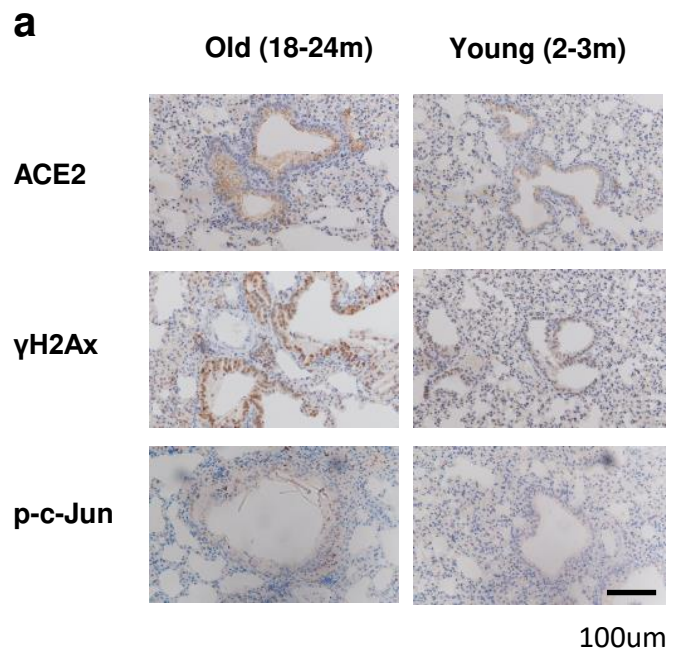

b

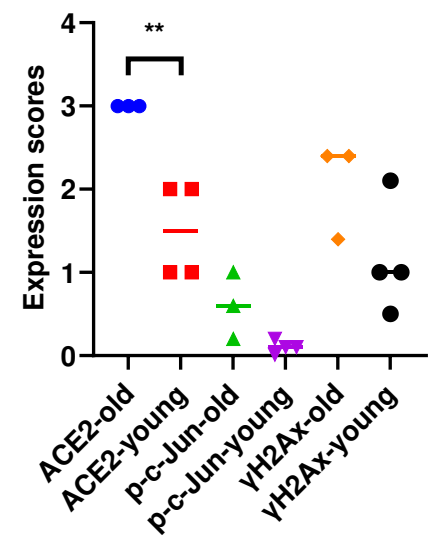

Fig. S5. Increased ACE2 expression in old mouse bronchial epithelial cells. Expression of ACE2, p-c-Jun and $\mathrm{yH} 2 \mathrm{Ax}$ in old $(18-24 \mathrm{~m}, \mathrm{n}=3)$ and young $(2-3 \mathrm{~m}, \mathrm{n}=4)$ mouse lung tissues. Representative immunohistochemical staining of ACE2, p-c-Jun and $\mathrm{yH} 2 \mathrm{Ax}$ is shown in (a). Scale bar: $100 \mu \mathrm{m}$. ${ }^{* *} \mathrm{p}<0.01$. 
Fig. 4

a
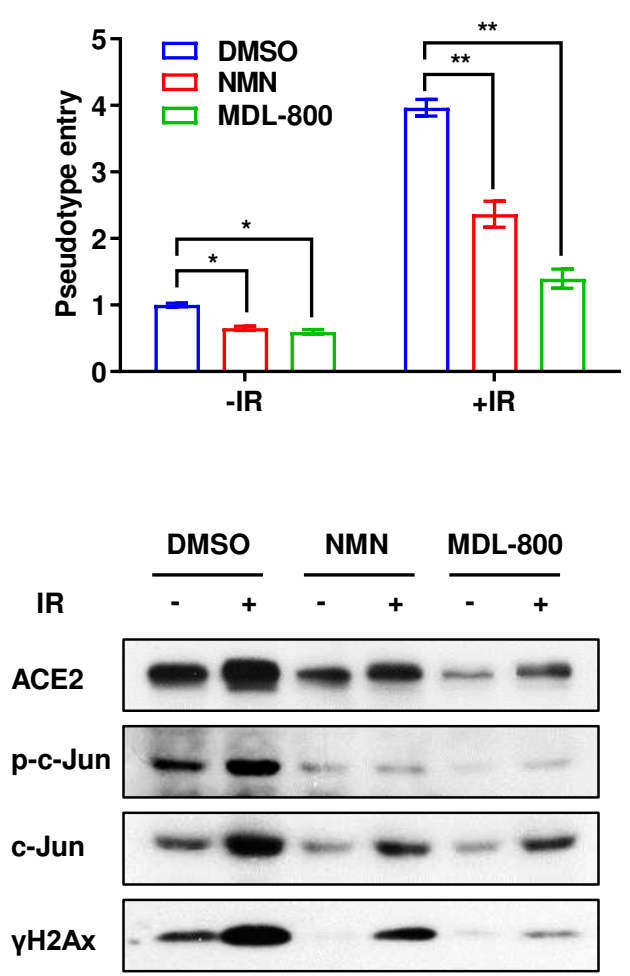

Actin

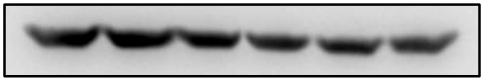

b

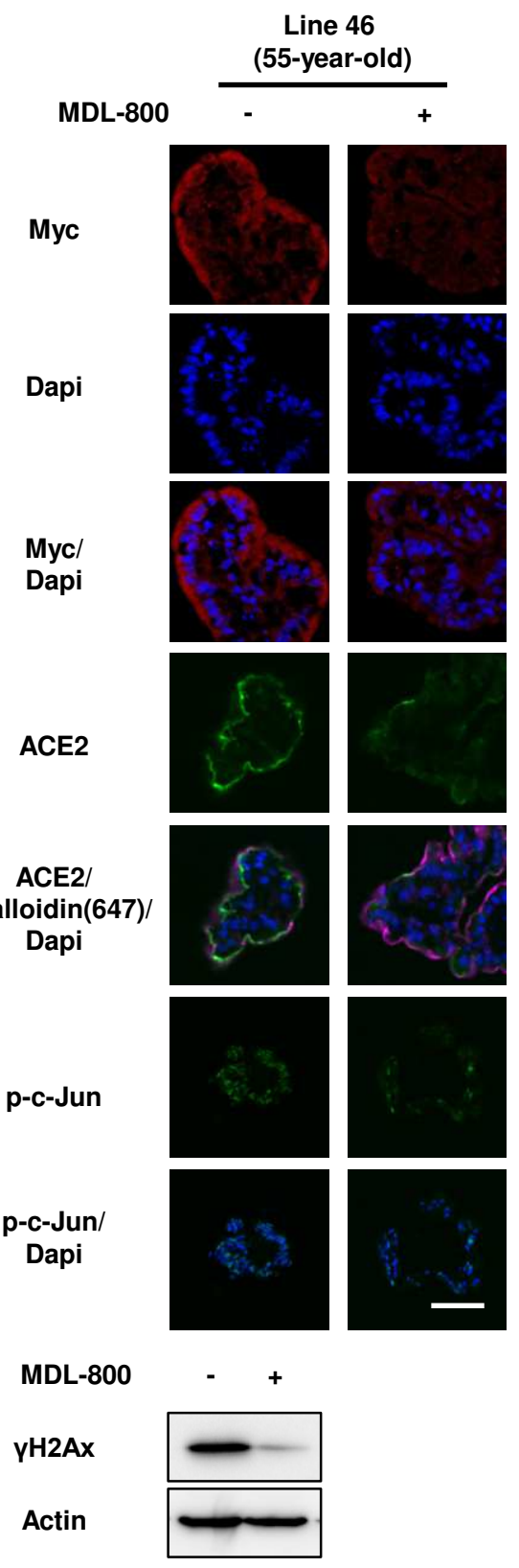

Fig. 4. Targeting DNA damage alleviates cell susceptibility to SARS-CoV-2 infection. a, Caco-2 cells were treated with NMN (1 mM) or MDL-800 $(20 \mu \mathrm{M})$ for 7 days and then treated with or without 10Gy IR. Three days later, the cells were incubated with the SARS-CoV-2 pseudovirus. Viral entry was analyzed by luciferase activity at $72 \mathrm{~h}$ after infection and expression of the indicated proteins was analyzed by western blot. $\mathbf{b}$, Differentiated organoids (Line 46) treated with MDL-800 $(20 \mu \mathrm{M})$ for 3 days were infected with SARS-CoV-2 pseudovirus harbouring Myc-EGFP. The organoids were harvested for immunostaining and western blotting with the indicated antibodies at 3 days postinfection. The EGFP signals were too weak to observe without immunostaining with Myc antibody. Scale bar, $50 \mu \mathrm{m}$. ${ }^{*} p<0.05,{ }^{* *} p<0.01$. 


\section{Materials and Methods}

\section{Constructs and plasmids}

Plasmids encoding SARS-CoV S glycoprotein (AAP13567.1), SARS-CoV-2 S glycoprotein (QHD43416.1) and MERS-CoV S glycoprotein (AFS88936.1) were constructed by inserting DNA fragments encoding codon-optimized SARS-CoV S protein lacking the last 19 amino acids (aa), encoding codon-optimized SARS-CoV-2 S protein lacking the last 19 amino acids (aa), MERS-CoV S protein lacking the last 16 aa into pCDNA3.0 between KpnI and EcoRI sites, respectively (1). The VSV-G encoding plasmid and lentiviral packaging plasmids pLP1 and pLP2 were obtained from Addgene (Cambridge, MA). Renilla-luciferase (RLuc) lentivirus expression vector pHHLVX-EF1 $\alpha$-Rluc-puro was purchased from HedgehogBio Science and Technology Ltd. The lentiviral plasmid expressing Myc-EGFP (pCDH-Myc-EGFP) was constructed by inserting DNA fragment encoding Myc-EGFP into pCDH-EF1MCS-T2A-Puro (SBI, CD510A-1) between NheI and NotI sites. The lentiviral reporter plasmid that expresses Firefly luciferase (pCDH-Luc) was constructed by inserting DNA fragment encoding Firefly luciferase into pCDH-CMV-MCS-EF1-Puro (SBI, CD511B-1) between NheI and BamHI sites. The lentiviral plasmids Lenticas9-Blast (\#52962) and Lentiguide-Puro (\#52963) were obtained from Addgene. The lentiviral plasmids encoding deletion of the POT1-binding recruitment domain (244-337aa) of TPP1 (TPP1 $\Delta \mathrm{RD})$ and lacking the first OB fold domain (1-126aa) of POT1 (POT1 $\triangle \mathrm{OB})$ were constructed by inserting DNA fragments with a Flag tag at the $\mathrm{N}$ terminus into pCDH-EF1-MCS-T2A-Puro (SBI, CD510A-1) between NheI and NotI sites. All primers and codon-optimized gene sequences used in this study are listed in Supplementary Table 2 .

\section{siRNAs, antibodies and reagents}

The siRNAs targeting human genes were obtained from Invitrogen and the target sequences were contained in Supplementary Table 3. Antibodies used in this manuscript were listed in Supplementary Table 5. Small molecular compounds caffeine was 
obtained from sigma, KU55933, VE-822, NMN and MDL-800 were obtained from Selleck, etoposide was obtained from sigma.

\section{Viruses, cell culture and transfection}

As previously described (2), authentic SARS-CoV-2 virus was isolated from the lung lavage fluid of an infected patient. All work with infectious SARS-CoV-2 was performed in the biosafety level 3 (BSL-3) facility of Beijing Institute of Microbiology and Epidemiology, Academy of Military Medical Sciences, China. Human embryonic kidney HEK293T cells, human lung adenocarcinoma Calu-3 cells, human colorectal adenocarcinoma Caco-2 cells were purchased from American Type Culture Collection (ATCC), and have been tested for mycoplasma contamination previously. Cells were routinely cultured in DMEM (Invitrogen) containing 10\% FBS (ExCell Bio). PEI and RNAiMax reagents (Invitrogen) were used for plasmid and siRNA transfection according to the manufacturer's instructions.

\section{TERC knockout Calu-3 cells}

TERC knockout Calu-3 cells were generated by CRISPR/Cas9. Calu-3-Cas9 stable cells were constructed by transfection with lentivirus expressing Cas9, which was packaged in 293T cells by co-expression of lentiCas9-Blast (Addgene \#52962), psPAX2 (Addgene \#12260) and pCMV-VSV-G (Addgene \#8454). The sgRNA (single guide RNA) sequence were cloned into the lentiGuide-Puro (Addgene \#52963) and packaged into lentivirus in $293 \mathrm{~T}$ cells, followed by transfection into Calu-3-Cas9 cells. sgRNA sequences targeting TERC gene and its promoter region were listed in Supplementary Table 2. After selection with puromycin, monoclonal cells were selected, cultured for approximately 45 passages and collected for genomic DNA extraction and amplification by PCR with the following primers: Forward, 5'GTCCTTAAGATTAATAATGTAGTAGTTACACTTGATTAAAGCCA -3'; Reverse, 5'- GCATGTGTGAGCCGAGTCCT -3'. The PCR products were sequenced.

\section{Telomerase Repeat Amplification Protocol (TRAP) assay}


The Telomerase Repeat Amplification Protocol (TRAP) assay was performed to determine telomerase activity by using TRAPeze Telomerase Detection kit (Millipore) as previously described (3). Briefly, cultured cell pellets were lysed with $1 \times$ CHAPS lysis buffer for $30 \mathrm{~min}$ on ice. After centrifugation at $12000 \mathrm{rpm}$ for $20 \mathrm{~min}$, the concentration of supernatant proteins was determined by Bio-Rad Protein Assay Kit. Twenty to fifty nanograms of cultured cell lysates were used for TRAP assays. The final reaction products were mixed with GelRed loading buffer (Generay Biotech) and run on a $10 \%$ polyacrylamide gel. The gel was exposed by Gel Image System (Tanon).

\section{Telomere length measurement}

The telomere length was measured according to the published method (4). Genomic DNA was isolated from cells using KingFisher Flex DNA purification instrument (ThermoFisher) with MagMAX ${ }^{\mathrm{TM}}$ DNA Multi-Sample Ultra 2.0 Kit (ThermoFisher). The primers for telomere PCR were tel1b: 5-CGGTTT(GTTTGG) ${ }_{5}$ GTT-3, used at a final concentration of $300 \mathrm{nM}$, tel2b: 5-GGCTTG(CCTTAC) $)_{5} \mathrm{CCT}-3$, used at a final concentration of 300nM. The primers for single-copy gene (36B4) PCR were 36B4u: 5-CAGCAAGTGGGAAGGTGTAATCC-3, used at a final concentration of 300nM, 36B4d: 5-CCCATTCTATCATCAACGGGTACAA-3, used at a final concentration of 500nM. $2 \times$ Mix (Qiagen) was used in qPCR reaction mixture with 9.2ng genomic DNA in each tube. qPCR was carried out on CFX-96 qPCR instrument (Bio-Rad). For telomere (T) PCR, procedure includes $95^{\circ} \mathrm{C}$ for $10 \mathrm{~min}$ and 20 cycles of $95^{\circ} \mathrm{C}$ for $15 \mathrm{~s}$, $56^{\circ} \mathrm{C}$ for $1 \mathrm{~min}$. For $36 \mathrm{~B} 4(\mathrm{~S}) \mathrm{PCR}$, procedure includes $95^{\circ} \mathrm{C}$ for $10 \mathrm{~min}$ and 30 cycles of $95^{\circ} \mathrm{C}$ for $15 \mathrm{~s}, 60^{\circ} \mathrm{C}$ for $1 \mathrm{~min}$. Relative $\mathrm{T} / \mathrm{S}$ ratio of each sample was calculated and reflected relative telomere length. The T/S ratio for each sample was measured twice.

\section{Packaging, concentration and titration of lentivirus and pseudotyped lentivirus}

HEK293T cells were cultured in growth media (10\% FCS in DMEM) supplemented with L-Glutamine until $70 \%$ confluent on a T-75 cell culture flask. Cells were then transfected with PEI reagent according to the manufacturer's instructions. Briefly, 20 $\mu \mathrm{g}$ plasmids containing PLP1 $(5.88 \mu \mathrm{g})$, PLP2 $(2.8 \mu \mathrm{g})$, VSV-G $(3.92 \mu \mathrm{g})$ and pCDH- 
Myc-EGFP $(7 \mu \mathrm{g})$, or pCDH-Luc $(7 \mu \mathrm{g})$, or pHHLVX-EF1 $\alpha$-Rluc-puro $(7 \mu \mathrm{g})$ were mixed with $58.8 \mu$ PEI reagent in $1.5 \mathrm{ml}$ DMEM without FCS and left for 15 minutes at room temperature. For packaging of pseudotyped lentivirus, VSV-G was substituted by SARS-CoV S, SARS-CoV-2 S or MERS-CoV S. The plasmid-PEI mixture was then added to the cells and the cell medium was changed 6h later. Forty-eight hours after transfection, the medium was collected, centrifuged at $1000 \mathrm{x}$ g for 5 minutes to remove cell debris, filtered through a $0.45 \mu \mathrm{m}$ filter and stored at $4^{\circ} \mathrm{C}$. Add another $15 \mathrm{ml}$ fresh medium into the T-75 cell culture flask and medium were collected $24 \mathrm{~h}$ later. After centrifugation and filter, the medium was combined with the medium stored at $4{ }^{\circ} \mathrm{C}$ and mixed with $5 \times$ lentivirus concentration solution (50\% PEG-8000, 1.5M Nacl). After incubation at $4^{\circ} \mathrm{C}$ overnight, the mix were centrifuged at $2000 \mathrm{xg}$ for 30 minutes at $4^{\circ} \mathrm{C}$. Carefully remove supernatant and resuspend the viral pellet with 500ul PBS. To remove serum proteins, the concentrated viral particles were centrifuged at $12000 \mathrm{x}$ g for 5 minutes at $4{ }^{\circ} \mathrm{C}$ and supernatant was aliquot and stored at $-80{ }^{\circ} \mathrm{C}$ until use. Titration of lentivirus were performed by qRT-PCR measurement of viral RNA using Lenti-pac HIV qRT-PCR Titration Kits (GeneCopoeia) kit according to the manufacturer's instructions. Briefly, lentivirus RNA was purified using TRIzol reagent and treated with DNase I to remove lentiviral expression plasmids. cDNAs were reverse transcribed using random primers and subjected to qPCR for detection of WPRE sequence of lentivirus. Plasmid containing WPRE sequence was gradient diluted and included in each PCR run so that the physical titer of lentivirus could be determined relative to the plasmid DNA sample by the standard curve method. Functional titer of lentivirus was obtained approximately by divide physical titer with 100 (5).

\section{Establishment, maintenance, differentiation and virus infection of intestinal organoid}

The ethics of the study was approved by the Institutional Review Board of Chinese PLA General Hospital (the reference number is S2020-013-01). For organoid establishment, surgically resected healthy small intestinal tissues were obtained from 2 donors (organoid termed line 0 was isolated from a 41-day-old male neonate with necrotizing 
enterocolitis, organoid termed line 46 was isolated from a 55-year-old woman with stomach cancer). The establishment, maintenance and differentiation of intestinal organoid were performed mainly according to the standard protocol established by Hans Clevers in 2011 (6). Briefly, the intestinal tissues were washed and chopped into smallest pierces. After incubated with Gentle Cell Dissociation Reagent (GCDR, STEMCELL), the tissue sections were pipetted up and down in DMEM with BSA to remove crypts from tissue. Isolated crypts were counted, mixed with Matrigel (Cornine) and plated on a 24-well plate. After the Matrigel dome was polymerized, $300 \mu 1$ of organoid growth medium (OGM, STEMCELL, components of the medium were illustrated in Supplementary Table 4) were added and changed every 2 days. Passage the organoid every 7-10 days by solute Matrigel dome with CRS (Corning), disruption the organoid by pipetting up and down vigorously, and re-plate the organoid with Matrigel. To differentiate organoid, organoid cultured in expansion medium for 7 days were subsequently cultured in organoid differentiation medium (ODM, STEMCELL, components of the medium were illustrated in Supplementary Table 4) for another 5-8 days. To detect the effect of IR and caffeine on SRAS-CoV-2 pseudovirus infection (Fig. 1D), organoids were cultured in ODM for 2 days and then treated with IR (10Gy) or caffeine $(1 \mathrm{mM})$. At $24 \mathrm{~h}$ post IR, organoids were flipped "inside-out" in suspension culture with ODM for 2 days to take the apical side of the cells at the outside of the spherical organoid (7) and then incubated with SARS-CoV-2 pseudovirus at approximately $\mathrm{MOI}=10$ for $3 \mathrm{~h}$ and then re-plated in Matrigel dome. Western blot and immunostaining were performed at 3 days after infection. To detect the effect of MDL800 on SRAS-CoV-2 pseudovirus infection (Fig. 4B, C), organoids were cultured in ODM for differentiation with or without MDL-800 for 3 days. Then the organoids were flipped, infected with SARS-CoV-2 pseudovirus and analyzed as above mentioned.

\section{Virus infection in cells}

For infection of pseudotyped virus in Caco-2 and Calu-3 cells, cultured cells were dissociated with trypsin and cell number was counted. After cells were seeded in a 24well plate, pseudotyped virus were added to the suspension cell at $\mathrm{MOI}=0.5$ and mixed. 
Virus entry were analyzed at 3 days after virus infection. For infection of authentic SARS-CoV-2 virus, adherent Caco-2 cells were incubated with SARS-CoV-2 virus at $\mathrm{MOI}=0.1$ for $1 \mathrm{~h}$, and then culture medium was replaced with fresh medium and virus entry were analyzed at $5 \mathrm{~h}$ after virus infection.

\section{Immunofluorescence of cells and organoids}

Cell immunofluorescence was performed as previously described (3). Briefly, cells grown on glass coverslips were fixed with 4\% PFA (paraformaldehyde), permeabilized with permeabilizing solution $(0.5 \%$ Triton-X100, $1 \%$ normal goat serum in PBS) and blocked in blocking solution ( $1 \%$ normal goat serum in PBS). The coverslips were then incubated with primary antibodies diluted with blocking solution. Primary antibodies used were illustrated in Supplementary Table 5. After washed in blocking solution for 3 times, coverslips were incubated with corresponding secondary antibodies. After washed in PBS for 3 times, nuclei were counterstained with 4',6-diamidino-2phenylindole (DAPI). For in combination with Tel-FISH, after the first detecting antibody staining in the IF protocol, cells were treated by 4\% PFA fixing and PBS washing and incubated with Alexa fluor 488-OO-(CCCTAAA) 3 PNA probe (catalog no. PNA1006, Applied Biosystems) for telomeric DNA hybridization by the manufacturer protocol. To detect protein expression and distribution in intestinal organoid, organoid was fixed with 4\% PFA for 30 min, washed 3 times with PBS, and dehydration with $20 \%$ sucrose in PBS overnight. Organoids were then flash frozen in optimal cutting temperature (OCT) and were cut to $10 \mu \mathrm{m}$ thick sections using a cryotome. Then, the slides were washed in PBS to remove OCT and permeabilized. The following procedures were the same as cell immunofluorescence. Confocal images were collected using a LSM880 confocal microscope (Zeiss).

\section{Senescence associated $\beta$-gal (SA- $\beta$-gal) staining}

The SA- $\beta$-gal staining was carried out according to the manufacturer's instructions (Cell Signaling). Briefly, Cells were washed once with PBS, fixed with $0.5 \%$ glutaraldehyde in PBS for 15 min. After washed in PBS for 3 times, cells were stained 
in X-gal solution (100 mM sodium phosphate, $2 \mathrm{mM} \mathrm{MgCl} 2,150 \mathrm{mM} \mathrm{NaCl}, 0.01 \%$ sodium deoxycholate, $0.02 \% \mathrm{NP}-40,5 \mathrm{mM}$ potassium ferricyanide, $5 \mathrm{mM}$ potassium ferrocyanide, $1 \mathrm{mg} / \mathrm{ml} \mathrm{X-gal} \mathrm{at} \mathrm{pH} 6.0) 6-24 \mathrm{~h}$ at $37^{\circ} \mathrm{C}$.

\section{qRT-PCR detection of gene expression and authentic SARS-CoV-2 entry}

Total cell RNA was extracted using TRIzol reagent according to the manufacturer's instructions (Invitrogen). To detect relative levels of authentic SARS-CoV-2 virus, cells infected with SARS-CoV-2 were performed with viral RNA extraction with QIAamp Viral RNA Mini Kit (52906, Qiagen) according to the manufacturer's instructions. First strand cDNA was reverse transcribed using PrimeScript RT reagent Kit with gDNA Eraser (Takara) and applied for qPCR amplification using TB Green ${ }^{\circledR}$ Premix Ex Taq ${ }^{\text {TM }}$ II (Takara) with the following primers: human ACE2 forward, 5'AGACCAAAGCATCAAAGTGAGGAT-3'; human ACE2 reverse, 5'TTAAAGGAGATTCTTGGTTTCAAATTAGCC-3'; human $\beta$-actin forward, 5'AGAAGAGCTACGAGCTGCCTGA-3'; human $\beta$-actin reverse, 5'CAATGATCTTGATCTTCATTGTGCT-3'; human DPP4 forward, 5'AAGTGGCGTGTTCAAGTGTG-3'; human DPP4 reverse, 5'ATGGTCAAGGTTGTCTTCTGG-3'; human IFN-beta forward, 5'AATTGAATGGGAGGCTTGAATACTGCCTCAAGG-3'; human IFN-beta reverse, 5'-GTCTCATTCCAGCCAGTGCTAGATGAATCTTGT-3'; human ISG15 forward, 5'-ATGTCGGTGTCAGAGCTGAAGGCG-3'; human ISG15 reverse, 5'CTTGTTATTCCTCACCAGGATGCTCAGAGGTT-3'; human OAS1 forward, 5'CAGCAACTCTGCATCTACTGGAC-3'; human OAS1 reverse, 5'TCAGCCTCTTGTGCCAGCTGC-3'. SARS-CoV-2 N forward, 5'GGGGAACTTCTCCTGCTAGAAT-3'; SARS-CoV-2 N reverse, 5'CAGACATTTTGCTCTCAAGCTG -3'. The relative expression was calculated by the comparative $\mathrm{Ct}$ method.

\section{Chromatin Immunoprecipitation (ChIP)}

ChIP assay was performed using EZ-Magna ChIP A/G Chromatin Immunoprecipitation 
Kit (Millipore) according to the manufacturer's instructions. Briefly, cells treated with or without IR were cross-linked with $1 \%$ formaldehyde for $10 \mathrm{~min}$, treated with glycine at a final concentration of $0.125 \mathrm{M}$ for $5 \mathrm{~min}$ at room temperature, and lysed in lysis buffer for $15 \mathrm{~min}$ on ice. Nuclei were resuspended in nuclei lysis buffer, and the crosslinked DNA was sonicated for $10 \mathrm{~min}$ (with a $5 \mathrm{~s}$ on/off cycle) using a sonicator (SONICS). The supernatant was used for immunoprecipitation with anti-p-c-Jun or IgG and DNA purified from input or immunoprecipitants were subjected to qPCR. The following primers were used in qPCR reaction: c-Jun-pro-1: 5'TGATCCTCTGTAGCCATGGGATCACA-3', c-Jun-pro-2: 5'TCCTCTCTTTGATCTGTGGCACTC-3'; c-Jun-dis-1: 5'TACCATTTGACCCAGCAATCC-3', c-Jun-dis-2: 5'TGCTATTGTGAATAGTGCTGCA-3'; c-Fos-pro-1: 5'GAGCAGTTCCCGTCAATCC-3', c-Fos-pro-2: 5'- GTGAGCATTTCGCAGTTCCT$3^{\prime}$.

\section{Animals}

$\mathrm{BALB} / \mathrm{c}$ female mice were obtained from Vital River and old mice were cultured under SPF conditions until 18-24 month. All experiments were carried out in accordance with the Guide for the Care and Use of Laboratory Animals published by the United States National Institutes of Health after securing the approval of the Committee of Animal Care of the Beijing Institute of Biotechnology.

\section{Clinical samples and immunohistochemistry}

Healthy intestinal tissues obtained from Chinese PLA General Hospital were isolated from patients of stomach cancers or right-sided colon cancers. The ethics of the study was approved by the Institutional Review Board of Chinese PLA General Hospital (the reference number is S2020-013-01). Immunohistochemical staining was performed as described previously (3). Briefly, the formalin-fixed paraffin sections were deparaffinized, rehydrated, and pretreated with 3\% $\mathrm{H} 2 \mathrm{O} 2$ for $25 \mathrm{~min}$. The antibodybinding epitopes of the antigens were retrieved by microwave treatment, and the 
sections were then preincubated with 3\% BSA to block nonspecific binding. The slides were then incubated with primary antibodies and subsequent secondary antibodies. The resource and dilution of primary antibodies used in the study were listed in Supplementary Table 5. Each specimen was assigned a score generated by multiplying the intensity of the staining (no staining $=0$; weak staining $=1$, moderate staining $=2$, strong staining $=3)$ by the percentage of stained cells $(0 \%-100 \%)$. The optimal cutoff value of the IHC scores were estimated using receiver operating characteristic (ROC) curve analysis.

\section{Single-Cell Analysis}

Single cell data of nasal ciliated 2 cells (8) were obtained from https://www.covid19cellatlas.org/index.healthy.html. Nasal data was extracted by using scanpy (v1.5.1) read_h5ad function and Seurat (v.3.1) was used for next step of analysis. Firstly, the data from different samples were integrated by IntegrateData function, and the cells were annotated according to the classification results in the previous article. Secondly, the cells were divided into two categories, ACE2+ group and ACE2- group, according to the presence or absence of ACE2 expression. Differential expression genes (logfc.threshold $=0.18)$ between ACE2+ cells versus ACE2- cells were performed enrichment analysis with Pathway Interaction Database (PID). The illustrations and statistical analysis were performed with R.

\section{Statistical analysis}

Statistical significance in cell susceptibility to pseudotyped virus or authentic virus, gene expression levels and gene enrichment in ChIP assay were assessed by multiple $t$ tests. The correlation of ACE2, p-c-Jun and $\gamma \mathrm{H} 2 \mathrm{Ax}$ was determined using Pearson correlation coefficient. All statistical tests were two-sided. Statistical calculations were performed using PRISM9. In all assays, $\mathrm{p}<0.05$ was considered statistically significant. 
References

1. Ou X, Liu Y, Lei X, Li P, Mi D, Ren L, et al. Characterization of spike glycoprotein of SARS-CoV-2 on virus entry and its immune cross-reactivity with SARS-CoV. Nature communications. 2020;11(1):1620.

2. Chi X, Yan R, Zhang J, Zhang G, Zhang Y, Hao M, et al. A neutralizing human antibody binds to the N-terminal domain of the Spike protein of SARS-CoV-2. Science. 2020;369(6504):650-5.

3. Cheng L, Yuan B, Ying S, Niu C, Mai H, Guan X, et al. PES1 is a critical component of telomerase assembly and regulates cellular senescence. Sci Adv. 2019;5(5):eaav1090.

4. Cawthon RM. Telomere measurement by quantitative PCR. Nucleic Acids Res. 2002;30(10):e47.

5. Geraerts M, Willems S, Baekelandt V, Debyser Z, Gijsbers R. Comparison of lentiviral vector titration methods. BMC Biotechnol. 2006;6:34.

6. Sato T, Stange DE, Ferrante M, Vries RG, Van Es JH, Van den Brink S, et al. Longterm expansion of epithelial organoids from human colon, adenoma, adenocarcinoma, and Barrett's epithelium. Gastroenterology. 2011;141(5):1762-72.

7. Co JY, Margalef-Català M, Li X, Mah AT, Kuo CJ, Monack DM, et al. Controlling Epithelial Polarity: A Human Enteroid Model for Host-Pathogen Interactions. Cell reports. 2019;26(9):2509-20.e4.

8. Vieira Braga FA, Kar G, Berg M, Carpaij OA, Polanski K, Simon LM, et al. A cellular census of human lungs identifies novel cell states in health and in asthma. Nat Med. 2019;25(7):1153-63. 
A

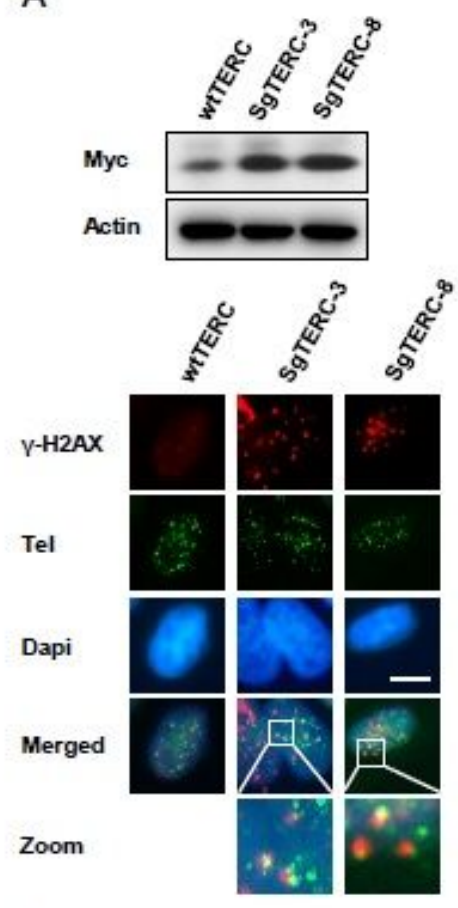

D
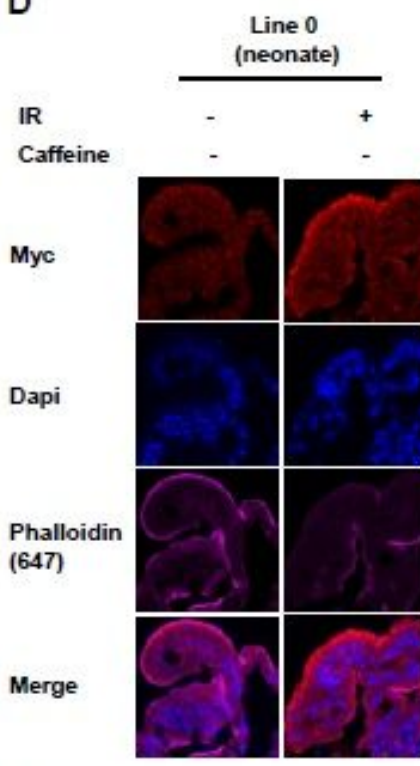

IR

Caffeine

$\mathrm{yH} 2 \mathrm{Ax}$

Actin
B
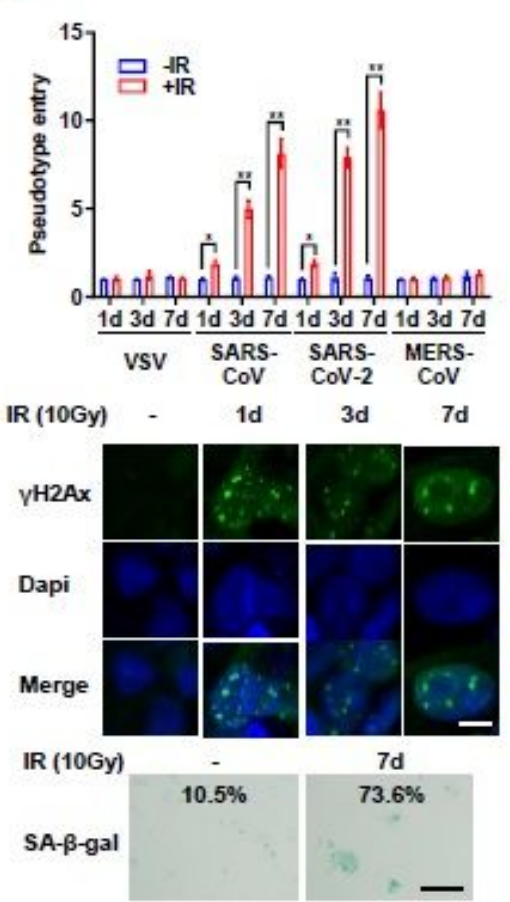

E
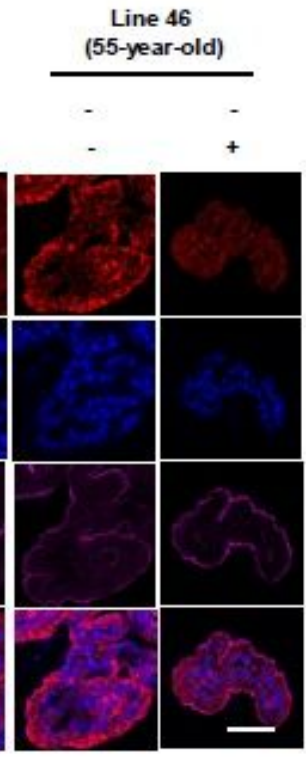

C
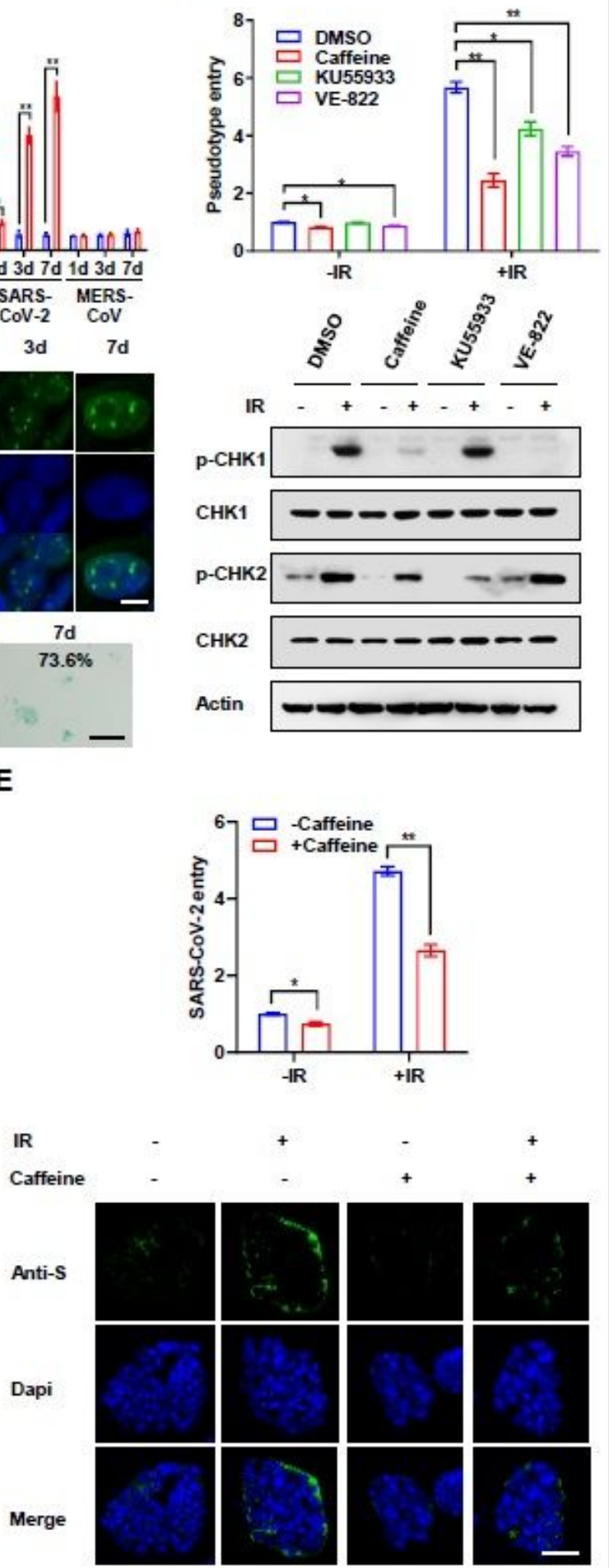

\section{Figure 1}

DNA damage promotes SARS CoV 2 entry a Monoclonal Calu 3 cells stably expressing sgTERC were infected with SARS CoV 2 pseudovirus harbouring Myc EGFP Virus entry was analyzed by western blot at 3 days after infection using the indicated antibodies Telomere damage was detected by immunostaining 
with an anti $\mathrm{yH} 2 \mathrm{Ax}$ antibody, followed by Tel Fish Scale bar, $10 \mu \mathrm{m}$ b, Calu 3 Rluc cells at various days after 10 Gy IR treatment were infected with pseudotyped viruses harbouring a firefly luciferase reporter gene Viral entry was calculated by dividing firefly luciferase activity by renilla luciferase activity at 3 days after infection DNA damage was detected by immunostaining with an anti $\mathrm{\gamma H} 2 \mathrm{Ax}$ antibody Scale bar, 10 $\mu \mathrm{m}$ SA $\beta$ gal staining was performed, and the results were quantified from 5 independent cell counts up to a total of at least 200 cells and are presented as the mean percentage of positive cells Scale bar, $100 \mu \mathrm{m}$ c, Caco 2 Rluc cells were pre treated with caffeine $1 \mathrm{mM}$ ), KU $5593310 \mu \mathrm{M}$ and VE $822100 \mathrm{nM}$ for $12 \mathrm{~h}$ and then subjected to IR treatment $10 \mathrm{~Gy}$ ) Cells were infected with SARS CoV 2 pseudovirus and analyzed as ( $d$ Differentiated organoids isolated from a neonate (Line 0 and a 55 year old woman (Line 46 were treated with IR $10 \mathrm{~Gy}$ ) or caffeine $1 \mathrm{mM}$ ) and then infected with SARS CoV 2 pseudovirus containing Myc EGFP Organoids were immunostained or detected by western blot using indicated antibodies at 3 day after infection The EGFP signal was too weak to observe without immunostaining with Myc antibody Scale bar, $50 \mu \mathrm{m}$ e, Caco 2 cells treated with caffeine $1 \mathrm{mM}$ ) and IR $10 \mathrm{~Gy}$ ), were incubated with authentic SARS CoV 2 virus for $6 \mathrm{~h}$ The relative virus levels were detected by qRT PCR analysis of SARS CoV $2 \mathrm{~N}$ protein or immunostaining of SARS CoV $2 \mathrm{~S}$ protein Scale bar, $50 \mu \mathrm{m}$ All data are represented as the mean SD of three (a c) or two (independent experiments p 005 p 001 p 0001. 


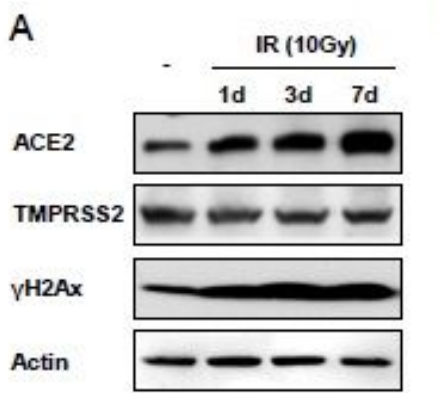

D

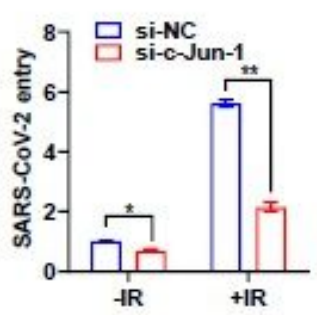

G

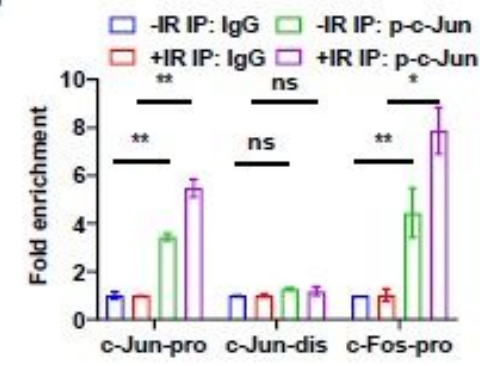

H
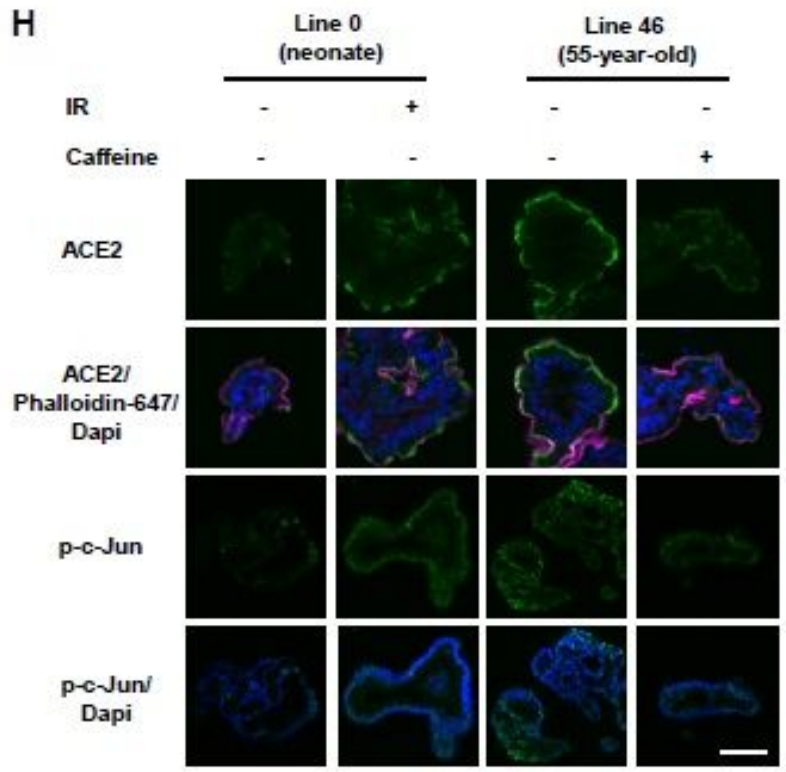

C

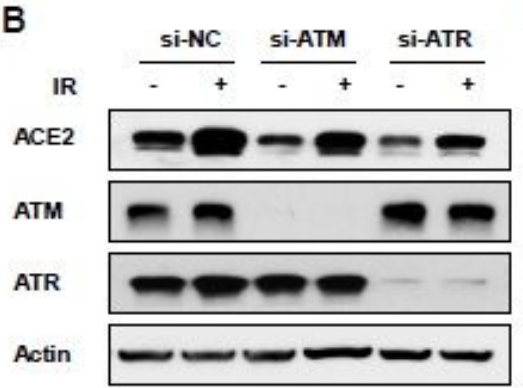

E

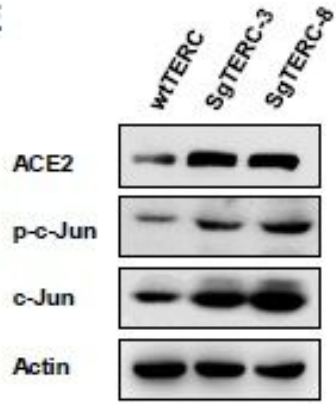

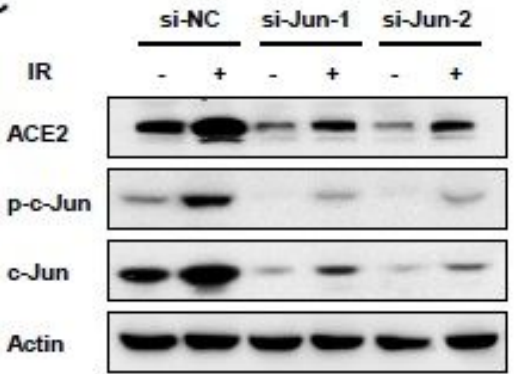

F

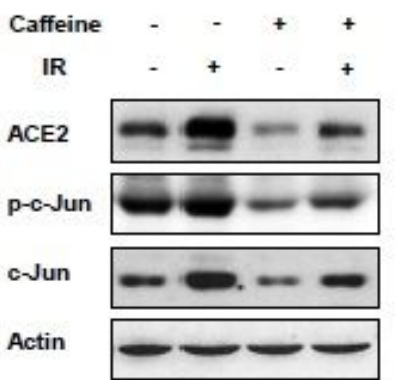

$-\mathbb{R}$
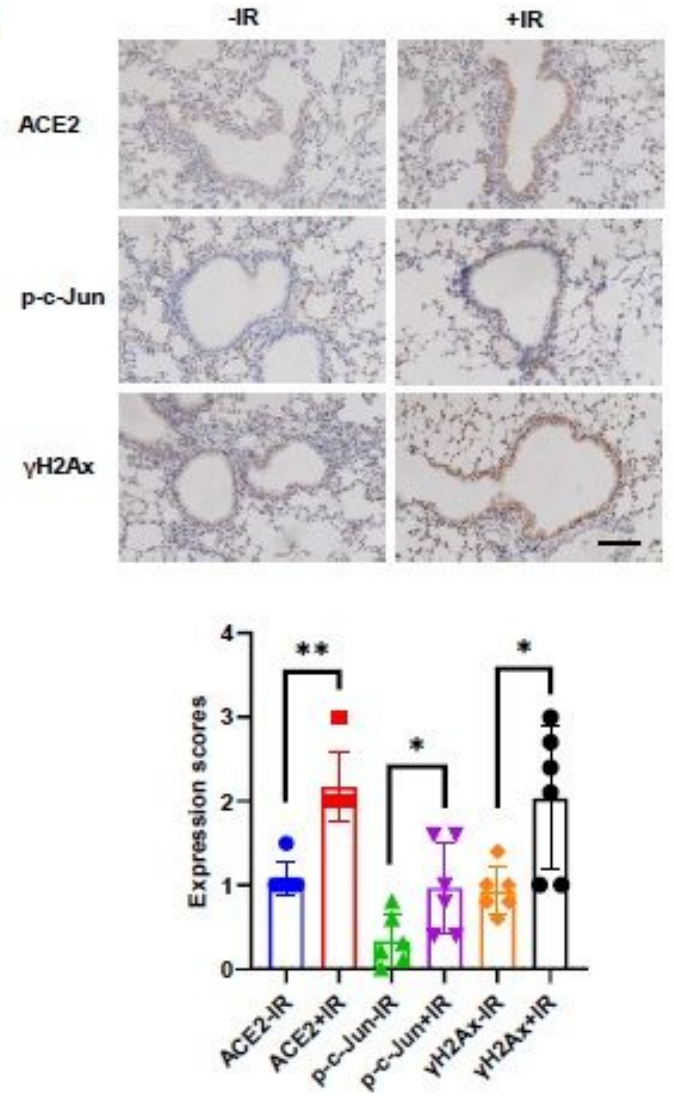

Figure 2

DNA damage enhances ACE 2 expression by activating $c$ Jun a b, Calu 3 Rluc cells at various days ( or Caco 2 cells transfected with ATM or ATR siRNA at 3 days ( after $10 \mathrm{~Gy}$ IR treatment were subjected to western blot analysis using the indicated antibodies c, Caco 2 cells after transfection with c Jun siRNA were treated as in $b$ and the expression of indicated genes were detected d, Caco 2 cells were transfected with c Jun siRNA $48 \mathrm{~h}$ prior to infection with authentic SARS CoV 2 virus for $6 \mathrm{~h}$ and then subjected to 
qRT PCR analysis of the relative viral entry e, Caco 2 cells were pre treated with caffeine $1 \mathrm{mM}$ ) for $12 \mathrm{~h}$ and then subjected to IR $10 \mathrm{~Gy}$ ) Western blot analysis was performed using the indicated antibodies at 3 days after IR $\mathrm{f}$, Monoclonal Calu 3 cells stably expressing sgTERC were subjected to western blot analysis using indicated antibodies $\mathrm{g}$, ChIP analysis of the occupancy of $\mathrm{p} c$ Jun on the indicated AP 1 binding site of the c Jun promoter (c Jun pro) and distal regions (c Jun dis) in Caco 2 cells at $3 \mathrm{~h}$ after $10 \mathrm{~Gy}$ IR treatment The $\mathrm{c}$ Fos promoter containing AP 1 binding site was included as a positive control lgG, normal serum Data shown are the mean SD of triplicate measurements from three independent experiments $h$, Differentiated organoids treated with IR $10 \mathrm{~Gy}$ ) or caffeine $1 \mathrm{mM}$ ) were immunostained with the indicated antibodies Scale bar, $50 \mu \mathrm{m}$ i Expression of ACE 2 p c Jun and $\mathrm{yH} 2$ Ax in 8 week old mice 3 days after treated with ( 6 or without ( 610 Gy IR Representative immunohistochemical staining of ACE 2 p c Jun and $\mathrm{yH} 2 \mathrm{Ax}$ was shown in the upper panel Scale bar, $100 \mu \mathrm{m} \mathrm{p} 005 \mathrm{p} 001$ 
A

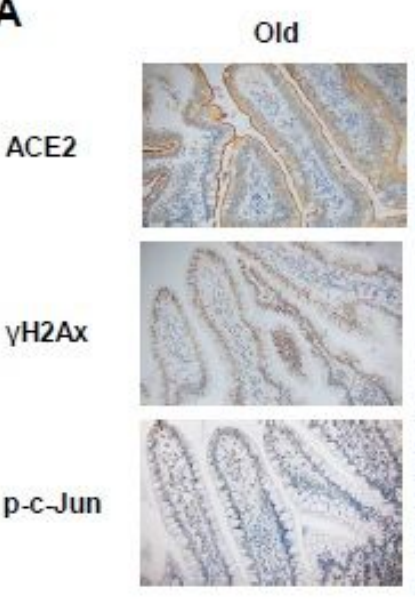

Young

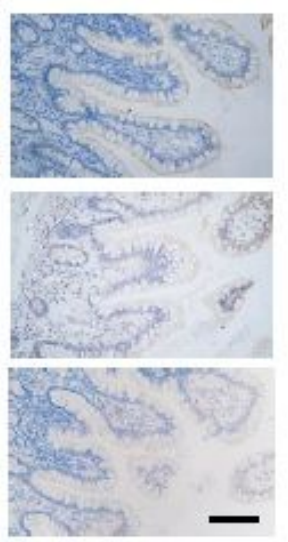

B

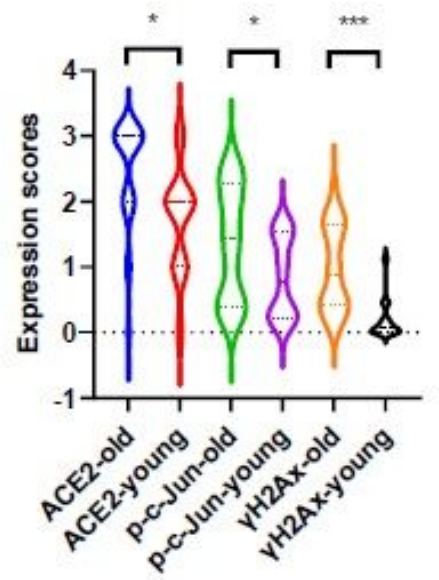

E
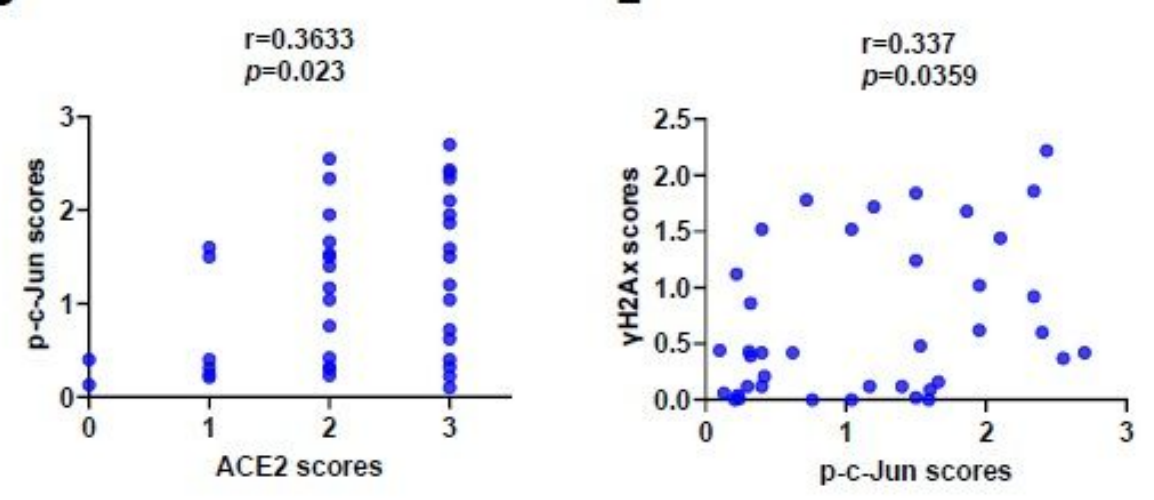

C

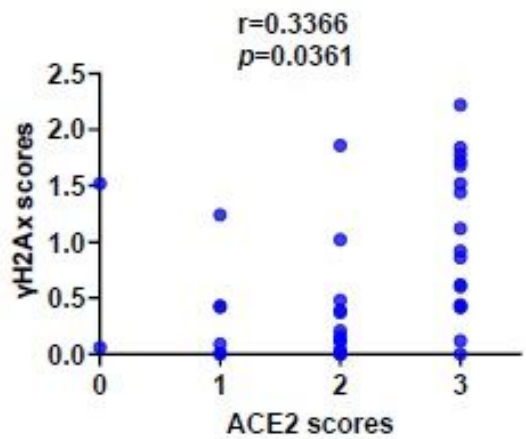

F

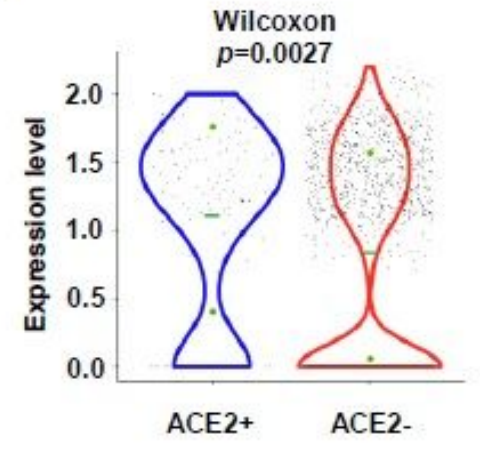

G

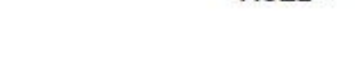


A
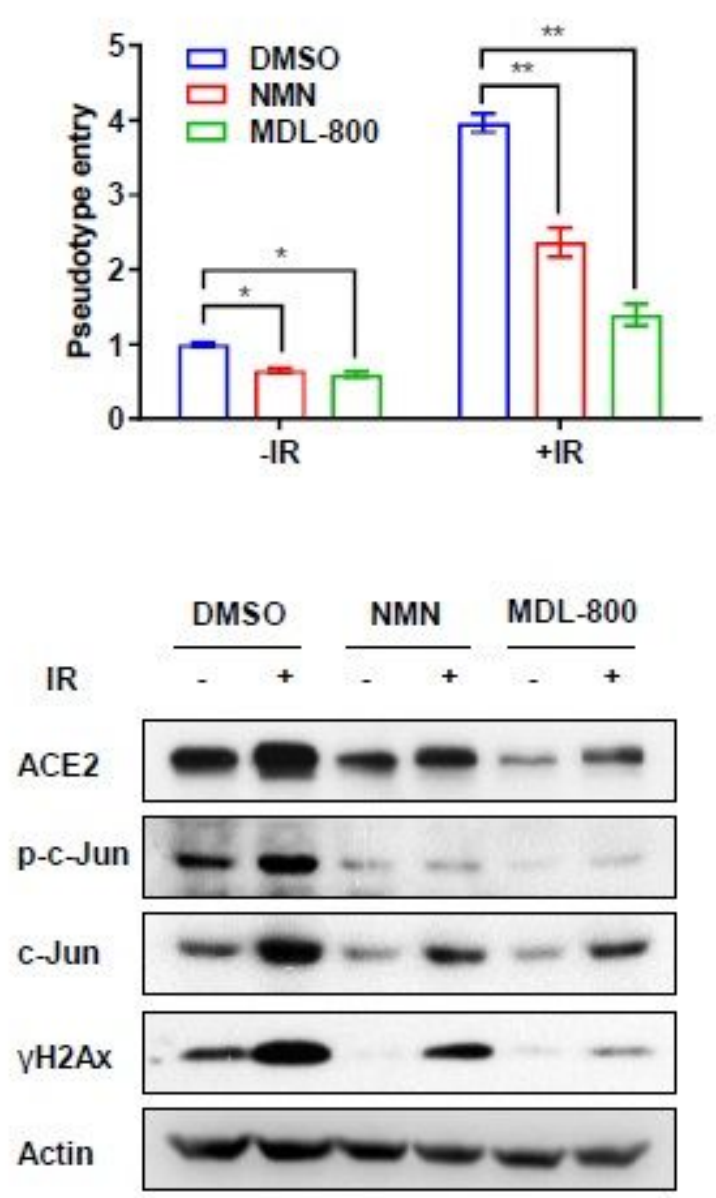

B
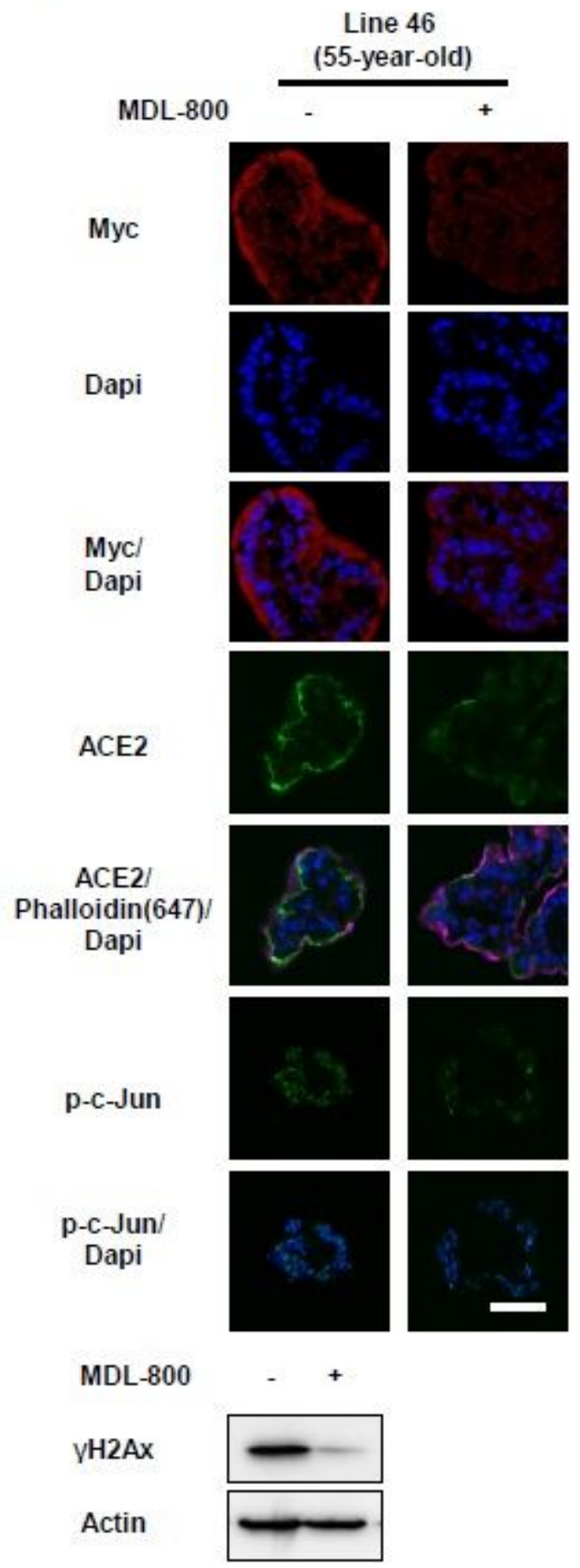

\section{Figure 4}

Targeting DNA damage alleviates cell susceptibility to SARS-CoV-2 infection. a, Caco-2 cells were treated with NMN $(1 \mathrm{mM})$ or MDL-800 $(20 \mu \mathrm{M})$ for 7 days and then treated with or without 10Gy IR. Three days later, the cells were incubated with the SARS-CoV-2 pseudovirus. Viral entry was analyzed by luciferase 
activity at $72 \mathrm{~h}$ after infection and expression of the indicated proteins was analyzed by western blot. b, Differentiated organoids (Line 46) treated with MDL-800 (20 $\mu \mathrm{M})$ for 3 days were infected with SARSCoV-2 pseudovirus harbouring Myc-EGFP. The organoids were harvested for immunostaining and western blotting with the indicated antibodies at 3 days postinfection. The EGFP signals were too weak to observe without immunostaining with Myc antibody. Scale bar, $50 \mu \mathrm{m}$. ${ }^{\star} \mathrm{p}<0.05,{ }^{\star *} \mathrm{p}<0.01$.

\section{Supplementary Files}

This is a list of supplementary files associated with this preprint. Click to download.

- supplementalTable1.xlsx

- supplementalTable2.xlsx

- supplementalTable3.xlsx

- supplementalTable4.xIsx

- supplementalTable5.xlsx 